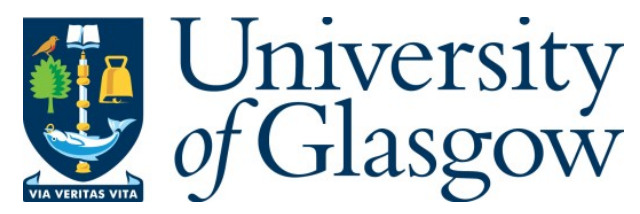

Tang, W., Shakir, M. Z., Imran, M. A., Tafazolli, R., Qaraqe, K. A., and Wang, J. (2016) Spectral and energy efficient cognitive radio-aided heterogeneous cellular network with uplink power adaptation. Wireless Communications and Mobile Computing, 16(14), pp. 2144-2162.

There may be differences between this version and the published version. You are advised to consult the publisher's version if you wish to cite from it.

This is the peer reviewed version of the following article: Tang, W., Shakir, M. Z., Imran, M. A., Tafazolli, R., Qaraqe, K. A., and Wang, J. (2016) Spectral and energy efficient cognitive radio-aided heterogeneous cellular network with uplink power adaptation. Wireless Communications and Mobile Computing, 16(14), pp. 2144-2162, which has been published in final form at http://dx.doi.org/10.1002/wcm.2673. This article may be used for non-commercial purposes in accordance with Wiley Terms and Conditions for Self-Archiving.

http://eprints.gla.ac.uk/132547/

Deposited on: 20 December 2016

Enlighten - Research publications by members of the University of Glasgow http://eprints.gla.ac.uk 


\title{
Spectral and Energy Efficient Cognitive Radio Aided Heterogeneous Cellular Network with Uplink Power Adaptation
}

\author{
Wuchen Tang, Muhammad Z. Shakir, Muhammad A. Imran, Rahim Tafazolli, \\ Khalid A. Qaraqe and Jiasong Wang
}

\begin{abstract}
In future heterogeneous cellular networks (HCN), cognitive radio (CR) compatible with device to device communication (D2D) technique can be an aid to further enhance system spectral and energy efficiency. The unlicensed smart devices (SDs) are allowed to detect the available licensed spectrum and utilise the spectrum resource which is detected as not being used by the licensed users (LUs). In this work, we propose such a system and provide comprehensive analysis of the effect of selection of SDs' frame structure on the energy efficiency, throughput and interference. Moreover, uplink power control strategy is also considered where the LUs and SDs adapt the transmit power based on the distance from their reference receivers. The optimal frame structure with power control is investigated under high SNR and low SNR network environments. The impact of power control and optimal sensing time and frame length, on the achievable energy efficiency, throughput and interference are illustrated and analysed by simulation results. It has been also shown that the optimal sensing time and frame length which maximizes the energy efficiency of SDs strictly depends on the power control factor employed in the underlying network such that the considered power control strategy may decrease the energy efficiency of SDs under very low SNR regime.
\end{abstract}

Index Terms-Cognitive radio; energy efficiency; device to device Communications; heterogeneous cellular network; optimal frame structure and uplink power control.

\section{INTRODUCTION}

Radio spectrum has been considered as one of the important limited resources as growing of wireless communication technology. However, recent reports by Federal Communications Commission (FCC) has shown that $70 \%$ of the allocated spectrum bands in US are not fully utilized,

W. Tang and M. A. Imran and R. Tafazolli are with Institute for Communication Systems (ICS, formerly CCSR), University of Surrey, Guildford, Surrey, United Kingdom, GU2 7XH, email: twc_wuchen@hotmail.com, $\{$ m.imran, r.tafazolli\}@ surrey.ac.uk.

M. Z. Shakir is with Systems and Computer Engineering Dept., Carleton University, Ottawa, Canada, email: muhammad.shakir@sce.carleton.ca.

K. A. Qaraqe is with the Electrical and Computer Engineering Dept., Texas A\&M University at Qatar, Doha, Qatar, email: khalid.qaraqe@qatar.tamu.edu.

J. Wang is with State Key Laboratory of Astronautic Dynamics, China Xi' an Satellite Control Center, 710043, China, email: wangjiasong6@126.com.

Parts of this paper were made possible by State Key Laboratory of Astronautic Dynamics (open fund 2013ADL-DW0401). In addition, we also acknowledge support from ICS. The statements made herein are solely the responsibility of the author[s]. whereas only $2 \%$ of the spectrum are used in the US for any moment [1]. Under this motivation, cognitive radio (CR) emerges as a striking technology that aims to improve the the current severely under-utilized radio spectrum by allowing the unlicensed user to access the licensed frequency bands [2-4] in heterogeneous network. In a CR aided heterogeneous cellular network $(\mathrm{HCN})$, unlicensed users who establish device to device (D2D) communication by sensing spectrum to find spectrum opportunities as well as to avoid intolerable interference to the licensed user (LUs) are named as smart devices (SDs). Spectrum opportunities could be considered as those licensed but temporally or geographically unused spectrum. In this context, opportunistic spectrum access is considered based on a typical frame structure which comprises of sensing and data transmission slot. The sensing and data transmission slots are required to be coordinated in a unit frame which mandates to (i) reduce the energy consumption with targeted throughput and (ii) reduce the interference to the LUs. Generally speaking, the length of the sensing slot determines the accuracy of spectrum sensing detection such that the higher accuracy can be obtained by collecting sufficient number of samples during the sensing phase, i.e., increasing the sensing length [5-7]. To provide higher sensing accuracy, several spectrum sensing algorithms have been introduced and developed, such as the traditional energy detection and eigenvalue-based detection algorithms which are based on the eigenvalues of the received signal covariance matrix [5-10].

\section{A. Motivation}

Spectrum efficiency is an critical issue for wireless communication networks. CR technique was first introduced in 1999 by J. Mitola III [11] to improve the spectrum usage efficiency and cope with spectrum scarcity problem through dynamically detecting and re-allocating white spaces in licensed radio band to unlicensed users. Besides spectrum efficiency, another important issue for the sustainable development of CR technology is energy efficiency, which has been recently marked as one of the alarming bottleneck in the telecommunication growth paradigm mainly due to dramatically varying global climate [12] and slowly progressing battery technology [13]. High energy efficiency will be one of key requirements for practical wireless networks because 
optimizing the energy-efficiency of cognitive radio networks (CRNs) not only reduces environmental impact, but also cuts network cost to enable economical green CR [14]. Meanwhile, CR may cause extra energy consumption because it relies on new and extra technologies and algorithms such as dynamic spectrum sensing and allocation, which potentially counteract its advantages and impede the largescale deployment of $\mathrm{CR}$ in future $\mathrm{HCN}$. Therefore, green radio has been becoming equally or even more important in contrast to increase communication capacity and prompting new dimensions of research and standard development activities.

On the other hand, D2D communication is considered as another potential solution to be incorporated into future device centric networks, due to its benefits of high bit rates, low delays and low power consumptions. Recently some works started to cover this area. However, many challenges still remain to be overcome $[15,16]$ such as how to integrate D2D communication into the future $\mathrm{HCN}$ with affordable cost and acceptable complexity on each layer. In [17] it also had been raised that $\mathrm{CR}$ aided D2D communication is a potential feature that can promote efficient resource utilization and interference management among different types of users in cellular networks. In this context, it is of immense important for the future $\mathrm{HCN}$ designers to critically calibrate the energy efficiency of various deployment strategies along with the other key performance metrics such as throughput and interference, in CR aided HCN with D2D function enabled. Open issues concerning the technical and economical challenges, as well as possible solutions, need to be investigated.

\section{B. Background on Throughput for Cognitive Radio}

In conventional CRNs, LUs and unlicensed users are normally named as incumbent users and cognitive users, respectively. From the cognitive users' perspective, the lower probability of sensing errors mandates to improve the throughput of the cognitive user. Therefore, a tradeoff is required to be defined between the sensing length and throughput of the cognitive users based on the frame structure $[18,19]$. Following each sensing period, the cognitive transmission starts when the licensed channel is considered as idle by the cognitive user. Otherwise, the cognitive user has to wait until the next frame to sense the licensed channels again before any opportunistic usage of channels. In [19], the optimization of spectrum sensing length has been studied using the sensing-throughput tradeoff metric. Specifically, the paper studied the design of the sensing length to maximize the achievable throughput of a single channel CRN, under the constraint of the probability of detection. To provide better service for cognitive users, it is advisable to aggregate the perceived spectrum opportunities obtained through simultaneous sensing over multiple channels. In [20], the design of the sensing time has been investigated in order to maximize the average achievable throughput of the multiple channels in CRN without causing harmful interference to the incumbent users or exceeding the transmission power limit of the cognitive users. The optimal sensing length is identified for the above problem under average power constraint. As an extended work of [20], authors in [21] also studied the problem of designing the optimal sensing length that maximizes the throughput of a wideband sensing-based spectrum sharing CRN and a wideband opportunistic spectrum access CRN. Different from [20], in [21] the authors introduce an average interference power constraint in the wideband opportunistic spectrum access scheme (besides the average transmit power constraint), in order to effectively protect the incumbent users from harmful interference for the realistic scenario of imperfect sensing. Moreover, the effect of this constraint on the optimal sensing time has also been demonstrated in this paper. However, both [20] and [21] assume an ideal traffic activity of incumbent user, which is synchronized with the activities (sensing and data transmission) of cognitive user and is not practical. It can be seen that the designed optimal sensing strategies of works above are only from the throughput perspective and energy efficiency related issues are not considered.

Compared with sensing length, transmission duration length also impacts the extent of interference between the incumbent user and the cognitive user and determines the throughput of the cognitive user. With the same frame structure, [22] considered a CRN that a cognitive user makes opportunistic access to a spectrum band which is legally licensed to a incumbent user according to the sensing result. Based on the required sensing time and the traffic pattern of incumbent user, an optimal value for transmission duration of cognitive user has been determined such that the throughput of the unlicensed user is maximized. However, in [22], the data transmission length is optimized only with single incumbent user coexisting in the CRN. Moreover, the analysis is based on the assumption that the spectrum sensing by the cognitive user is perfect.

\section{Background on Energy Efficiency for Cognitive Radio}

Apparently the majority of the current research aims at improving the throughput of CRN and the research related to energy efficiency of cellular CRN is very limited. In [23], the authors proposed energy efficiency based transmission duration design and power allocation methods. However, the system model is very simple and the interference from incumbent user to cognitive user is ignored. Because the length of sensing slot is assumed fixed, the impact of the sensing length on the sensing accuracy is not considered. Moreover, the principle and the procedure of the employed power allocation method is not given. In [24], the optimal sensing strategy is studied based on sequential sensing over multiple channels. The sensing-access strategies and the sensing order is identified to achieve the maximum energy efficiency.

Besides, use of proper power control strategy in CRN could further improve energy efficiency and reduce interfer- 
ence to incumbent users and there are some related works on power control in CRNs. In [25], an opportunistic power control strategy for the cognitive user is proposed, which has been proved to be effective in the sense that it maximizes the achievable rate of cognitive user while guaranteeing the outage probability of the incumbent user not to be degraded. Similarly, with known channel primary radio link, a power control for the CR fading channel is studied in [26] to maximize its ergodic capacity subject to the cognitive user's transmit power constraint as well as the constraint on the maximum ergodic capacity loss of the primary radio link due to the CR transmission. In [27], the author considered the problem of maximizing the throughput of a CRN while protecting incumbent users of the spectrum. Two mixed distributed/centralized control schemes (for downlink and uplink scenarios) that require minimal cooperation between cognitive and primary devices are proposed. It has been shown that the algorithms result in significant performance gain, in terms of the downlink and uplink throughput of the cognitive network. Different from previous works, the aim of the analysis on the power control strategy for CRN in this work would not only on throughput or capacity, but mainly for energy efficiency enhancement in $\mathrm{HCN}$.

\section{This Work Contributions}

In this paper, a CR aided $\mathrm{HCN}$ is considered which consists of LUs and D2D enabled SDs. D2D enabled SDs are unlicensed and they are interfering or interfered with/by LUs. In other words, no specific spectrum band will be assigned for D2D communications and SDs only opportunistically utilize the licensed channels assigned to LUs. It is to note that the referred SD-users and "cognitive (secondary) user" both perform spectrum sensing but they are different in nature. Firstly, conventional cognitive users still belong to conventional cellular network and the only difference with incumbent (primary) users is that they only have secondary right to use the spectrum in condition that no intractable interference is caused. D2D is separate network but needs to be well integrated into cellular network. D2D and cellular network comprise our referred HCN. Besides opportunistically utilizing licensed spectrum, SDs may have their own allocated spectrum band for communication and this is negotiable with cellular hosts. In this work, we focus on the its performance with no dedicated spectrum available. Secondly, conventional secondary users would not distinguish between uplink and downlink spectrum resource they should access. In case that they target at a carrier in downlink resource, it is very likely that they will experience long waiting time due to many continuous sensing and longer silent periods, which cause severe delay and intolerable interference to user terminal of LUs' system if sense errors occur. Furthermore, in future cellular networks, users may not prefer to subscribe over secondary spectrum resources because QoS is hardly guaranteed and their throughput highly depends on the traffic load of primary users. On the contrary, D2D model is very flexible and efficient, which may allow users to experience benefits in terms of smaller communication latency, increased data rate and reduced energy consumption.

In this work, variable frame structure of SDs is considered with various sensing and data transmission lengths for spectrum detection and opportunistic D2D transmission, respectively. The energy efficiency problem is formulated as a function of variable frame structure. The SD performs energy detection for spectrum sensing and then is allowed for $\mathrm{D} 2 \mathrm{D}$ transmission on the licensed channel subject to the sensing results. The contributions of this work are described as:

- we propose a CR aided $\mathrm{HCN}$ and provide the analytical expression of energy efficiency and throughput for the SD and interference to the LU.

- we address the selection of optimal sensing and frame length of the SD cognitive transmission under high SNR and low SNR environments in CR aided HCN.

- we investigate the impact of power control (over the transmission links between LU and eNodeB and between SDs involved in D2D links) under the two SNR regimes mathematically on energy efficiency, which are also verified by simulation results.

- we study and compare the selection of the frame structure and power control factor subject to network environments and required system performance such as average received SNR and transmission delay tolerance.

The rest of this paper is organized as follows. Section II defines the system model and explains how the SD detects and accesses the licensed spectrum bands in a CR aided HCN. In Section III, we analyse and derive the expression of energy efficiency in our proposed CR aided HCN with power control. The analysis on throughput of the SD and interference to $\mathrm{LU}$ is also provided in this section. In Section IV, we formulate the energy efficiency and provide the mathematical analysis to study the impact of optimal sensing, frame length and power control. Simulation results and discussions to validate the analytical analysis are presented in Section V. Finally, we conclude the paper in Section VI.

\section{SYSTEM MODEL}

In this paper, we consider a $\mathrm{CR}$ aided $\mathrm{HCN}$ consisting of LUs and SDs, which is shown as Fig. 1. LUs perform conventional cellular communications by uplink and downlink via evolved Node Bs (eNBs) with Frequency division duplexing (FDD) scheme. To initialize a D2D link, SDs need to perform a cell search to build communication with eNB for registration. Then peer discovery and synchronization would be performed to establish a D2D link and they are always completed before SDs perform spectrum sensing. There are two schemes for the eNB to pair D2D devices and they are called as a-priori and a-posteriori, which was introduced in [28]. In a-priori schemes, the network (and/or the devices them- selves) detect SDs candidates prior to 


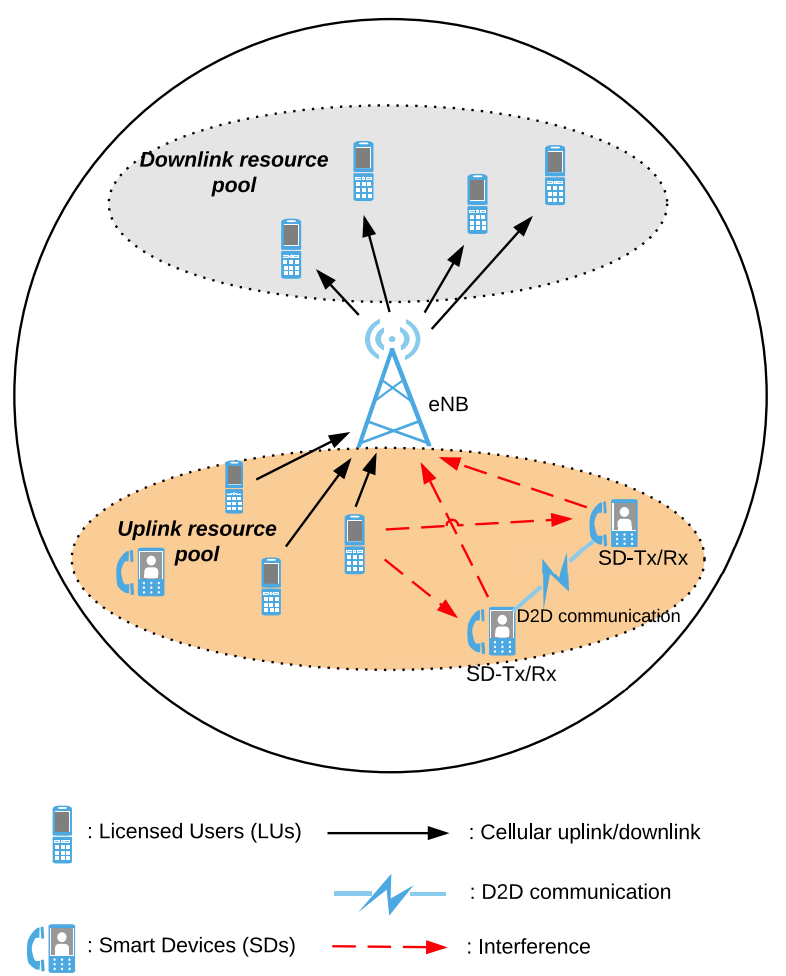

Fig. 1. Graphical illustration of CR aided HCN where SDs establish D2D comm opportunistically over uplink spectrum licensed to LU.

commencing a communication session between the devices while in a-posteriori device discovery, the eNB realizes that two communicating devices are in the proximity of one another and thereby they are D2D candidates when the communication session is already ongoing between the SDs. It is to note that in this work we assume that SDs are paired and synchronized by the former scheme (a-priori). All the necessary control signalling between SDs and eNB would be completed in the cell search and peer discovery phase such that the SDs are aware about who they are paired with and the targeted spectrum band to sense. In the meanwhile, the traffic patterns of LUs are also possible obtained by SDs, who are able to adjust their frame structure to achieve better performance.

The spectrum resources used for uplink and downlink by different LUs are determined and allocated by eNB. SDs perform D2D communication with uplink cellular spectrum resources because uplink spectral resource reusing is more desirable $[29,30]$. On the one hand, reusing uplink resources is more tractable than reusing downlinks as in the former case the potential victims of D2D communication are eNBs rather than user terminals, who can bear much less interference than eNBs. On the other hand, uplink traffic always tends to be less than downlink traffic. It is very likely that SDs could not access a targeted spectrum band due to heavy traffic of downlink channels. Besides, downlink resourcemap in current network such as LTE-A is very complicated due to many dedicated resources.

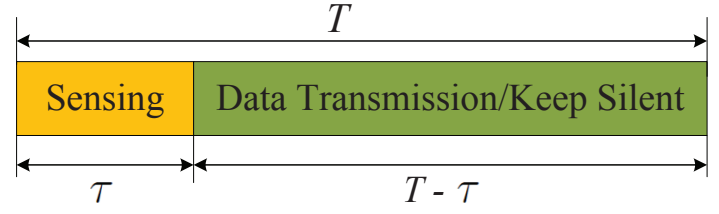

Fig. 2. A graphical structure of a typical frame structure of CR aided SD transmission.

The SD may be considered as a terminal with $M$ transmit antennas such that the $\mathrm{SD}$ collects $N$ samples from the LU during the sensing phase for each device. The collected samples will be forwarded to a fusion center for combined processing and decision. The SD performs spectrum sensing of the licensed frequency band by using the joint detector proposed in [31] to determine the status of each channel. Energy detection ${ }^{1}$ scheme is utilized for the joint spectrum sensing. The data transmission of the SD is activated subject to the spectrum sensing results based on the following two hypotheses for each channel

$$
\begin{aligned}
& \mathcal{H}_{0}: \boldsymbol{y}(n)=w(n), \\
& \mathcal{H}_{1}: \boldsymbol{y}(n)=h_{L S}(n) s(n)+w(n),
\end{aligned}
$$

where $\boldsymbol{y}(n)$ is the observed complex time series received at instant $n ; w(n)$ for all $n=\{1,2, \cdots, N\}$ represents an independent and identically distributed (i.i.d) circularly symmetric complex Gaussian (CSCG) with zero mean and $N_{0}$ variance. Hypothesis $\mathcal{H}_{0}$ and $\mathcal{H}_{1}$ stand for the spectrum band detected as idle and occupied, respectively. In (2), the vector $h_{L S}(n)$ typically represents the propagation channel between the corresponding LU and the SD and the signal $s(n)$ for all $n=\{1,2, \cdots, N\}$ denotes a standard scalar i.i.d random process and stands for the source signal to be detected. Once the channels has been confirmed as idle, the SD starts to perform D2D communication by transmitting over these channels. It is assumed that (i) the SD is heavily loaded and always has data to transmit, (ii) the traffic load of the LU is exponentially distributed with the mean of the occupied and the idle durations denoted by $\alpha_{1}$ and $\alpha_{0}$, respectively.

\section{A. Smart Device Frame Structure}

A typical frame structure of the SD is shown in Fig. 2 where each frame with length $T$ consists of the sensing slot with length $\tau$ and the data transmission slot with length

\footnotetext{
${ }^{1}$ In practice, SDs usually have no or limited knowledge about the LUs' signals and in this case energy detection is a reasonable option for spectrum sensing [32, 33]. Hence, An energy detection approach has been assumed in this paper for spectrum sensing because of its simplicity, ease of implementation, and low computational complexity [34]. Moreover, the aim of this paper is to characterize the impact of power control and optimal sensing time and frame length, on the achievable energy efficiency, throughput and interference on the proposed D2D aided heterogeneous wireless network without going into the details of complex spectrum sensing schemes. The more sophisticated techniques like match filter detection or cyclostationary feature detection can be used for signal classification if more a priori knowledge about the structure of the primary signal is available [11].
} 
$T-\tau$. The SD performs energy detection for spectrum sensing and then decide to transmit or keep silent according to the sensing result. It is worthy of note that the time for D2D signalling and link establish are not included in this frame structure because that it is assumed that these procedures has been finished in advance. In other words, SDs has been paired for D2D communications by eNB but the corresponding spectrum resources have not been allocated and thus need SDs to explore themselves since channel spectrum occupancy is very dynamic in such HCN environment. For any transmission on available channel, it may consist of a transmission link and two interference links and SD-Tx and SD-Rx denote the transmitter and receiver of the SD pair, respectively. The instantaneous channel gain of the D2D transmission link on the available channel is denoted as $h_{s s}$ while the interference link from LU to SD$\mathrm{Rx}$ and from SD-Tx to eNB are shown as the red arrows in Fig. 1, whose channel gain are denoted as $h_{L S}$ and $h_{s e}$, respectively. Channel information are assumed to be ergodic stationary and known before transmission phase and the quality of channel could be estimated by very short time compared with the sensing length. For example, in IEEE 802.11a, only 4 pilot symbols are used for channel estimation [35] while in IEEE 802.22 wireless regional area network (WRAN) thousands of samples are required for a typical sensing [36]. Therefore, for the frame structure of $\mathrm{SD}$, the channel estimation time could be ignored and thus is not considered in Fig. 2. The signals of LU are assumed to be complex-valued phase-shift keying (PSK) signals, whereas the noise at the SD is assumed to be independent and identically distributed circularly symmetric complex Gaussian (CSCG) with zero mean and $N_{0}$ variance.

It is to note that even if the channel is available when it is sensed, it is still possible that the corresponding LU activates during the data transmission phase of D2D communication between two SDs, which is illustrated by Fig. 3. This would lead to the situation that both the SD and the LU transmit on the same channel which not only decreases the throughput of SD but also introduces the interference to the LU. In addition, there may be an additional interference because of sensing errors of the spectrum, that is, the situation where the spectrum is actually occupied by the LUs and wrongly detected as idle which is referred to as missed detection and is illustrated by Fig. 4. Moreover, in contrast with missed detection, another type of sensing errors that is referred to as false alarm, describes the situation that the spectrum is actually idle and wrongly detected as occupied by the LUs, which would also decrease the throughput and energy efficiency of HCN. Therefore, the optimal sensing time should be addressed in order to provide sufficient sensing accuracy and save the time as much as possible which is supposed to be reserved for data transmission in a unit frame.

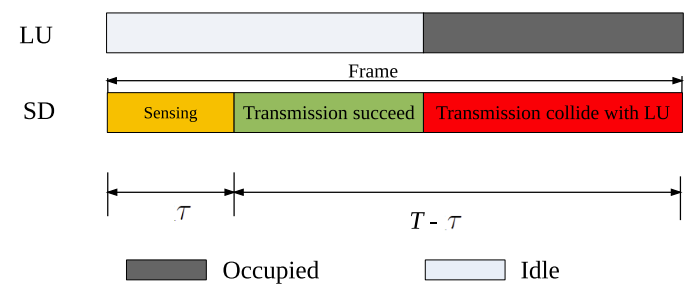

Fig. 3. A graphical illustration of data collision when LU becomes active in a typical cognitive radio transmission with perfect sensing.

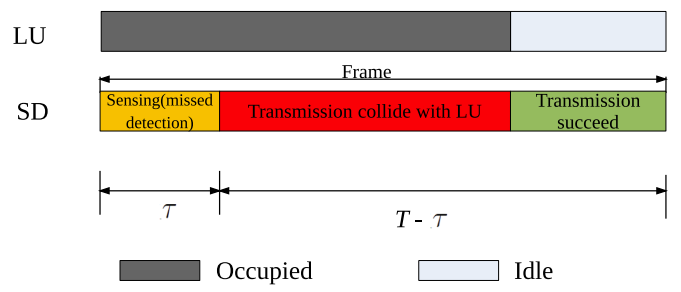

Fig. 4. A graphical illustration of data collision in a typical cognitive radio transmission with missed detection.

\section{B. Mobile User Distribution}

In this paper, we assume that all the mobile users including both the LU and SD are mutually independent and uniformly distributed in a $\mathrm{HCN}$. The probability density function (PDF) of the distribution of mobile users which are located at $\left(r_{d}, \theta_{d}\right)$ from its serving eNB and can be given in polar coordinate as

$$
p\left(r_{d}, \theta_{d}\right)=\frac{r_{d}}{\pi R_{m}^{2}}
$$

where $R_{m}$ is the radius of $\mathrm{HCN}$ cell, $0 \leq r_{d} \leq R_{m}$ and $0 \leq \theta \leq 2 \pi$.

\section{Propagation Model}

The radio environment of a typical wireless cellular network is described by: (i) distance dependent pass-loss, (ii) shadowing and (iii) multichannel fading. Path-loss is due to the decay of the intensity of a propagating radio-wave, and it requires an accurate estimation for proper determination of electric field strength, signal to noise ratio (SNR) [37]. In this work, to simulate a real channel environment, we consider a two slope path-loss model to obtain the mean received power as a function of distance between the mobile user and the respective serving eNB. It has been shown that two slope (or commonly known as dual-slope) path-loss model is suitable for strong line of sight $(\mathrm{LoS})$ conditions [35].

The dual-slope path-loss model consider two separate path-loss exponents, $\beta_{a}$ and $\beta_{b}$ which are referred to as basic and additional path-loss exponents, respectively. These pathloss exponents are used to characterize two different propagation environments, together with a breakpoint distance $g$ 
between them where propagation changes form one regime to the other. More explicitly, the signal attenuates with basic path-loss exponent $\beta_{a}$ before breakpoint and attenuates with additional path-loss exponent $\beta_{b}$ after breakpoint. Here, $g=\frac{4 h_{r x} h_{t x}}{\lambda_{c}}$ strictly depends on the antenna height of eNB (receiver in uplink) $h_{r x}[\mathrm{~m}]$, the antenna height of the mobile user (transmitter in uplink) $h_{t x}[\mathrm{~m}]$ and wavelength of the carrier frequency $\lambda_{c}$. With this dual-slope pathloss model, the average received signal power which is denoted as $P[\mathrm{~W}]$ at the reference receiver (eNB in case of communication with LUs and SD-Rx in case of D2D communication between SDs) from the desired mobile user with distance $r_{d}$ is given by [37]

$$
P=\frac{K}{r_{d}^{\beta_{a}}\left(1+r_{d} / g\right)^{\beta_{b}}} P^{t},
$$

where $K$ is the path-loss constant and $P^{t}[\mathrm{~W}]$ defines the mobile user transmit power. If power control is performed, all the mobile users (including LUs and SDs) are considered to be capable of adapting its transmit power automatically while maintaining a certain signal power received at the eNB. The uplink adaptive transmit power can be expressed as

$$
P^{t}=\min \left(P^{\max }, P_{0} \frac{r_{d}^{\beta_{a}}\left(1+r_{d} / g\right)^{\beta_{b}}}{K}\right),
$$

where $P_{0}[\mathrm{~W}]$ denotes the signal power received at the eNB, which is the cell specific parameter used to control the target signal to interference ratio. $P^{\max }$ is assumed maximum transmission power. Because the setting of $P_{0}$ determines both the achievable throughput of SD and the interference of LU, $P_{0}$ should be setted subject to the quality of service (QoS) requirements of specific networks considered. To set the target $P_{0}$ with fixed $P^{\max }$, we define $\Gamma$ and $\Gamma_{p}[\mathrm{~dB}]$ as the power control factor of SD and LU, respectively, which denote the difference between the corresponding required $P_{0}$ and $P^{\max }$. For example, if $\Gamma=-50 \mathrm{~dB}$, the power control of SD is set as $P_{0}=P^{\max }-50[\mathrm{~dB}]$. Note that (5) is the simplified version of conventional uplink power control which is recently approved by 3GPP in Long Term Evolution (LTE) networks [38].

\section{ENERGY EFFICIENCY OF D2D AIDED HCN}

In this work, the SD preforms energy detection spectrum sensing and transmit with adaptive power based on the decision made by the sensing phase. We assume the signal is complex-valued PSK modulated and noise is circularly symmetric complex Gaussian. Under energy detection scheme, the probability of detection and the probability of false alarm for the licensed channel can be expressed as a function of sensing length $\tau$ and energy detector decision threshold $\epsilon$, which had been derived in [19] as:

$$
\mathscr{P}_{d}(\tau, \epsilon)=\mathcal{Q}\left(\left(\frac{\epsilon}{N_{0}}-\gamma-1\right) \sqrt{\frac{\tau f_{s}}{2 \gamma_{+} 1}}\right),
$$

$$
\mathscr{P}_{f a, \epsilon}(\tau, \epsilon)=\left(\left(\frac{\epsilon}{N_{0}}-1\right) \sqrt{\tau f_{s}}\right),
$$

where $\gamma$ is the received SNR from the LU at the SD on the licensed channel, $f_{s}$ represents the sampling frequency and $\mathcal{Q}(\cdot)$ is the complementary distribution function of the standard Gaussian $\mathcal{Q}(x)=\frac{1}{\sqrt{2 \pi}} \int_{x}^{\infty} \exp \left(-\frac{t^{2}}{2}\right) d t$. It can be seen that both $\mathscr{P}_{d}$ and $\mathscr{P}_{f a}$ are related to the decision threshold $\epsilon$. Consequently, $\mathscr{P}_{d}$ and $\mathscr{P}_{f a}$ are also related to each other. For example, $\mathscr{P}_{f a}$ is always higher when a lower $\mathscr{P}_{d}$ is required and vice versa. To control the interference to LUs, the target detection probability $\overline{\mathscr{P}}_{d}$ should be guaranteed. With a given target detection probability $\overline{\mathscr{P}}_{d}$, (7) could be further expressed as

$$
\mathscr{P}_{f a}(\tau)=\mathcal{Q}\left(\sqrt{2 \gamma+1} \mathcal{Q}^{-1}\left(\overline{\mathscr{P}}_{d}\right)+\sqrt{\tau f_{s}} \gamma\right) .
$$

Then, if the licensed channel is detected as idle $\left(\mathcal{H}_{0}\right)$, the SD would perform data transmission during the data transmission slot, whereas if the licensed band is sensed as occupied $\left(\mathcal{H}_{1}\right)$, the $\mathrm{SD}$ has to keep silent until the next frame. In this section, we formulate the energy efficiency problem of the SD and study the frame structure optimization and power control, in order to maximize the energy efficiency with sufficient protection to LU and the targeted throughput of SD in the HCN. To highlight the the impact of power control, the energy efficiency problem would be formulated with power control compared with the case without power control.

With power control, both the SD and LU would always use the adaptive power (5), $P_{S D}^{t}$ and $P_{L U}^{t}$ to transmit, respectively. The instantaneous transmission rate of the SD on the licensed channel is denoted by $\hat{r}_{0}$ when the channel is actual idle $\left(\mathcal{H}_{0}\right)$, or denoted by $\hat{r}_{1}$ when the channel is actual occupied by $\operatorname{LU}\left(\mathcal{H}_{1}\right)$, which occurs when both the $\mathrm{SD}$ and the LU transmits on the same channel and interfere with each other. Here, $\hat{r}_{0}$ and $\hat{r}_{1}$ are respectively given by

$$
\begin{gathered}
\hat{r}_{0}=\log _{2}\left(1+\frac{h_{s s} P_{S D}^{t}}{N_{0}}\right)=\log _{2}\left(1+\frac{P_{S D}^{0}}{N_{0}}\right), \\
\hat{r}_{1}=\log _{2}\left(1+\frac{h_{s s} P_{S D}^{t}}{h_{L S} P_{L U}^{t}+N_{0}}\right)=\log _{2}\left(1+\frac{P_{S D}^{0}}{P_{L U}^{0}+N_{0}}\right),
\end{gathered}
$$

where $h_{s s}$ and $h_{L S}$ represent the channel gains from SDTx to SD-Rx and from LU to SD-Rx, respectively as we discussed before while $P_{S D}^{0}$ and $P_{L U}^{0}$ define the received power level of secondary signal and primary signal at secondary receiver, respectively.

As illustrated in Fig. 3, because LU may change its status anytime, it is possible that one LU suddenly becomes active but the corresponding licensed frequency band is still occupied by the SD, which brings interference to both the LU and the SD. We assume that the traffic loads of the LUs are exponentially distributed with the mean of the occupied and the idle durations denoted by $\alpha_{1}$ and $\alpha_{0}$, respectively. 


$$
\begin{aligned}
\hat{I} & =\frac{T-\tau}{T} \mathbb{E}\left\{P_{S D}^{t}\left(\mathscr{P}\left(\mathcal{H}_{0}\right) h_{s e}\left(1-\mathscr{P}_{f a}\right) \mathscr{P}_{p}^{s}+\mathscr{P}\left(\mathcal{H}_{1}\right) h_{s e}\left(1-\mathscr{P}_{d}\right) \mathscr{P}_{i p}^{s}\right)\right\}, \\
& =\frac{T-\tau}{T} \mathbb{E}\left\{P_{S D}^{0}\left(\mathscr{P}\left(\mathcal{H}_{0}\right)\left(1-\mathscr{P}_{f a}\right) \mathscr{P}_{p}^{s}+\mathscr{P}\left(\mathcal{H}_{1}\right)\left(1-\mathscr{P}_{d}\right) \mathscr{P}_{i p}^{s}\right)\right\} .
\end{aligned}
$$

In this case, the percentage of transmission with interference (collided transmission, red portion in Fig. 3) due to this LU unpredictable activity out of data transmission duration can be expressed as [39]

$\mathscr{P}_{p}^{s}=1-\frac{\alpha_{0}}{T-\tau}\left(1-\exp \left(-\frac{T-\tau}{\alpha_{0}}\right)\right), \quad T>\tau>0$.

It is to note that the interference above is because of the unpredictable activity of the LU rather than the sensing errors. In practice, spectrum sensing is always imperfect and the sensing errors due to missed detection lead to more interference between the SD and LU while false alarm would not cause further interference but will waste spectrum resource and thus decreases both energy efficiency and throughput of CRNs. In the condition that missed detection occurs, the percentage of transmission with interference (collided transmission, red portion in Fig. 4) out of data transmission duration is given by [39]:

$$
\mathscr{P}_{i p}^{s}=\frac{\alpha_{1}}{T-\tau}\left(1-\exp \left(-\frac{T-\tau}{\alpha_{1}}\right)\right), \quad T>\tau>0 .
$$

Based on the analysis above, the average interference to the LU and the average throughput of the SD could be expressed by (13) at top of this page and

$$
\begin{aligned}
\hat{R}= & \frac{T-\tau}{T} \mathbb{E}\left\{\mathscr{P}\left(\mathcal{H}_{0}\right)\left(1-\mathscr{P}_{f a}\right)\left(\hat{r}_{0}\left(1-\mathscr{P}_{p}^{s}\right)+\hat{r}_{1} \mathscr{P}_{p}^{s}\right)\right. \\
& \left.+\mathscr{P}\left(\mathcal{H}_{1}\right)\left(1-\mathscr{P}_{d}\right)\left(\hat{r}_{0}\left(1-\mathscr{P}_{i p}^{s}\right)+\hat{r}_{1} \mathscr{P}_{i p}^{s}\right)\right\}
\end{aligned}
$$

respectively. $\mathbb{E}\{\cdot\}$ represents the function of expectation and it is to note that expectation of a constant is equal to the constant itself.

In order to formulate the energy efficiency problem of $\mathrm{SD}$, the power consumed in a frame duration should be addressed, which mainly contains three parts: electronic circuit consumption $P_{c}$, spectrum sensing consumption $P_{s}$, and the data transmission consumption on the licensed channel $P_{S D}^{t}$. The electronic circuit power $P_{c}$ is the average power assumption of device electronics, such as mixers, filters, and digital to analog converters, which is almost fixed. The power used during spectrum sensing is much small compared to the data transmission power. The average total power consumed within a frame could be calculated as

$$
\hat{E}=\left\{\begin{array}{lll}
P_{c} T+P_{s} \tau, & \mathcal{H}_{1} \mid \mathcal{H}_{0} \text { or } \mathcal{H}_{1} \mid \mathcal{H}_{1} \\
P_{c} T+P_{s} \tau+P_{S D}^{t}(T-\tau), & \mathcal{H}_{0} \mid \mathcal{H}_{0} \text { or } \mathcal{H}_{0} \mid \mathcal{H}_{1}
\end{array}\right.
$$

which could be expressed using conditional probability theory as

$$
\begin{aligned}
\hat{E}= & P_{c} T+P_{s} \tau+P_{S D}^{t} \times \\
& (T-\tau)\left(\mathscr{P}\left(\mathcal{H}_{0}\right)\left(1-\mathscr{P}_{f a}\right)+\mathscr{P}\left(\mathcal{H}_{1}\right)\left(1-\overline{\mathscr{P}}_{d}\right)\right) .
\end{aligned}
$$

The energy efficiency of SDs in the proposed CR aided $\mathrm{HCN}$ is defined as

$$
\hat{J}=\frac{\text { Average Number of the Bits Transmitted }}{\text { Average Total Energy Consumed }}=\frac{\hat{R} T}{\hat{E}}
$$

which is measured in bits/Joule/Hz. By substituting (14) and (16) into (17), we have the energy efficiency of SD in the considered HCN with power control which could be expressed as (18).

Similarly, denote $r_{0}, r_{1}, I, R, E$ and $J$ as the instantaneous transmission rates of SD with LU coexisting and without LU coexisting, the average interference to the $\mathrm{LU}$, the average throughput of the SD, the average total power consumption within a frame and the energy efficiency of SD in the proposed CR aided HCN without power control, respectively. It is to note that in case that power control is not employed, SDs would use $P_{S D}^{\max }$ as transmission power and consequently the expressions of corresponding metrics can be obtained by simply replacing $P_{S D}^{t}$ by $P_{S D}^{\max }$ in the equations from (9) to (18).

\section{OPTIMIZATION OF ENERGY EFFICIENCY}

Based on the derived the energy efficiency formula for our proposed $\mathrm{CR}$ aided $\mathrm{HCN}$, the energy efficiency optimization problem, under the average interference constraint and total transmission power constraint can be formulated as follows

$$
\begin{array}{ll}
\text { maximize } & \hat{J} \\
\text { subject to } & \hat{I} \leq \overline{\hat{I}}, \hat{R} \geq \overline{\hat{R}}, T>\tau>0 .
\end{array}
$$

The $\overline{\hat{I}}$ is maximum acceptable interference for LU and $\bar{R}$ is the targeted throughput for the SD. In order to maximize an energy efficiency, a proper sensing time $\tau$ is key to achieve required sensing accuracy without unnecessary time waste. Frame length $T$ is also important to transmit more data as well as avoid transmission collision due to LUs' traffic. Besides, $P_{S D}^{t}$ dominants the total power consumption 


$$
\begin{gathered}
\hat{J}=\frac{(T-\tau) \mathbb{E}\left\{\mathscr{P}\left(\mathcal{H}_{0}\right)\left(1-\mathscr{P}_{f a}\right)\left(\hat{r}_{0}\left(1-\mathscr{P}_{p}^{s}\right)+\hat{r}_{1} \mathscr{P}_{p}^{s}\right)+\mathscr{P}\left(\mathcal{H}_{1}\right)\left(1-\mathscr{P}_{d}\right)\left(\hat{r}_{0}\left(1-\mathscr{P}_{i p}^{s}\right)+\hat{r}_{1} \mathscr{P}_{i p}^{s}\right)\right\}}{P_{c} T+P_{s} \tau+P_{S D}^{t}(T-\tau)\left(\mathscr{P}\left(\mathcal{H}_{0}\right)\left(1-\mathscr{P}_{f a}\right)+\mathscr{P}\left(\mathcal{H}_{1}\right)\left(1-\mathscr{P}_{d}\right)\right)} . \\
\tilde{J}=\frac{(T-\tau)\left(\mathscr{P}\left(\mathcal{H}_{0}\right)\left(1-\mathscr{P}_{f a}\right) r_{0}+\mathscr{P}\left(\mathcal{H}_{1}\right)\left(1-\mathscr{P}_{d}\right) r_{1}\right)}{P_{c} T+P_{s} \tau+P_{S D}^{\max }(T-\tau)\left(\mathscr{P}\left(\mathcal{H}_{0}\right)\left(1-\mathscr{P}_{f a}\right)+\mathscr{P}\left(\mathcal{H}_{1}\right)\left(1-\mathscr{P}_{d}\right)\right)}, \\
\tilde{\hat{J}}=\frac{(T-\tau)\left(\mathscr{P}\left(\mathcal{H}_{0}\right)\left(1-\mathscr{P}_{f a}\right) \hat{r}_{0}+\mathscr{P}\left(\mathcal{H}_{1}\right)\left(1-\mathscr{P}_{d}\right) \hat{r}_{1}\right)}{P_{c} T+P_{s} \tau+P_{S D}^{t}(T-\tau)\left(\mathscr{P}\left(\mathcal{H}_{0}\right)\left(1-\mathscr{P}_{f a}\right)+\mathscr{P}\left(\mathcal{H}_{1}\right)\left(1-\mathscr{P}_{d}\right)\right)} .
\end{gathered}
$$

and determines the achievable throughput so it also highly matters. These three variables jointly decide the result of (19). However, it can be seen that (19) is exceptionally complex with complicated expressions of $\hat{J}$ and $\hat{R}$. The constraints, $\overline{\hat{I}}, \overline{\hat{R}}$ are also not fixed due to different QoS of different wireless networks. In this section, we take insight into the concavity of the energy efficiency $\hat{J}$ and $\hat{R}$ to provide mathematical proof for the optimization results in simulation. Besides, the impact of power control on the achievable energy efficiency is analysed mathematically.

\section{A. Optimal Sensing and Frame Length}

To explore the impact of sensing and frame length on energy efficiency, we now look into the impact on throughput firstly which has simpler expression than energy efficiency. If we define $D=T-\tau$ which indicates the data transmission length, the expression of $\hat{R}$ (14), can be considered as function of $(\tau, D)$. It is to note that $\tau$ and $D$ are independent and if we replace $T-\tau$ by $D, \mathscr{P}_{p}^{s}$ and $P_{i p}^{s}$ are both as function of $D$ only and have nothing to do with $\tau$. In this case, according to the Theorem 1 of [18], one conclusion could be reached that there exists an optimal sensing time which yields the maximum achievable throughput and $\hat{R}$ is concave for the range of $\tau$ in which $\mathscr{P}_{f a} \leq 0.5$.

Proposition 1: $\hat{R}$ is increasing when $\mathrm{T}$ approaches $\tau$ and converge as $\mathrm{T}$ approach $+\infty$.

\section{Proof: See Appendix A.}

Apparently Proposition 1 is not sufficient to reach any conclusion about concavity of $\hat{R}$ but it is the most related mathematical evidence. Base on Proposition 1, the concavity of $\hat{R}$ can be discussed in term of its monotonicity. Specifically, $\hat{R}$ is concave if $\hat{R}$ monotonically increases and otherwise it is not concave. Moreover, the monotonicity of $\hat{R}$ depends on the other parameters in (14) such as transmission power such that concavity of $\hat{R}$ is not fixed with different network environments.

In the sequel, similar as $\hat{R}$, the convexity of energy efficiency, as $\hat{J}=\frac{\hat{R} T}{\hat{E}}$, with respect to $\tau$ and $T$ both depend on the value of other parameters in (18). The exhaustive search has to be employed for the optimal value of $\tau$ and $T$ for the maximum achievable energy efficiency.

\section{B. Impact of Power Control}

Based on the derived energy efficiency formula of proposed $\mathrm{HCN}$ with power control, in this part the impact of power control on the energy efficiency is investigated. To further simplify equation (18) and considering a common case that the status of LU is not changing very frequent, the interference brought by the asynchronous activities of LU and SD could be ignored, which means $\mathscr{P}_{p}^{s}=0$ and $\mathscr{P}_{i p}^{s}=1$. In this case, the simplified energy efficiency without and with power control would be approximately evaluated by (20) and (21), respectively.

Proposition 2: Denote $\Delta J=\widetilde{\hat{J}}-\widetilde{J}$ and consider $\Delta J=$ $f\left(P_{S D}^{t}\right)$ with $\operatorname{dom} f=\left\{P_{S D}^{t} \mid P_{S D}^{\max } \geq P_{S D}^{t}>0\right\}, \Delta J$ is not always positive as a function of $P_{S D}^{t}$.

\section{Proof: See Appendix B.}

Based on Proposition 2, one conclusion can be reached that power control by (4) may not be energy efficient when $P_{S D}^{t}$ is very small, which may occur in a very low SNR environment. Please note that the nature of power control by (4) is to reduce transmission power with required SNR achieved but in some extreme cases it is not beneficial for energy efficiency. Proposition 2 exams such an extreme case when $P_{S D}^{t}$ is very small. With the essence of Shannon channel capacity formula, it should be always more energy efficient with lower transmission power (lower SNR if same noise is considered). However, this only apply to the energy efficiency which is calculated as successfully transmitted information over received signal power. In order to calculate the accurate energy efficiency in practise, it can not be ignored that the impact of real channel propagation (pathloss and fading) on the transmission signal power, as well as an additional power consumption such as electronic circuit power and sensing power. Specifically, under very low SNR network, the transmission power is not dominant power consumption any more considering the total power consumed and in this case transmission with the maximum power is more energy efficient with controlled transmission power by (4). This is the reason why the power control by (4) may not be energy efficient for low SNR. Consequently, if power control like (4) is needed to be employed to 


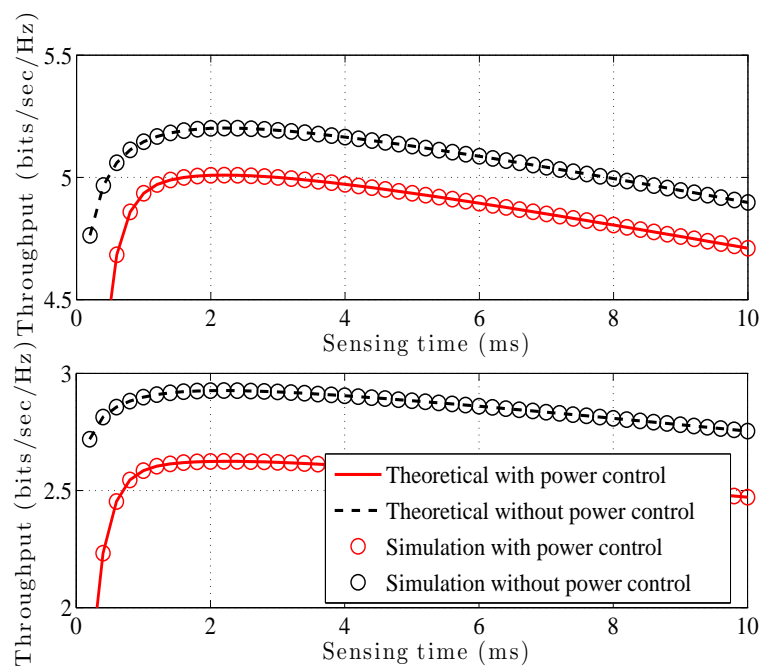

Fig. 5. Throughput vs sensing time with high SNR (upper) and low SNR (lower).

further improve energy efficiency, we may need to find the acceptable range of the received power to avoid low SNR regime which decreases energy efficiency.

\section{Simulation RESUlts}

In this section, we present and discuss the simulation results of the considered $\mathrm{HCN}$ with variable frame structure and power control strategy. Energy detection is employed for spectrum sensing. We assume that signal is complexvalued PSK modulated and noise is circularly symmetric complex Gaussian. As we discussed in the previous section, a two slope path-loss model is considered to simulate a real channel environment and all the LUs and SDs are randomly distributed in a $\mathrm{HCN}$ cell with radius $500 \mathrm{~m}$. It has been reported that the maximum transmission power of mobile terminal ranges from $30 \mathrm{~mW}$ to $2 \mathrm{~W}$ subject to different wireless networks [38, 40]. Due to the concern of human health and environment problem, it would be the common belief that transmission power of mobile terminals in future wireless communication system would be further less. Therefore, we consider two maximum transmission power of the SD with $P_{S D}^{\max }=800 \mathrm{~mW}$ and $P_{S D}^{\max }=50$ $\mathrm{mW}$, which could be considered as two SNR regimes: the high SNR and the low SNR circumstance, respectively. The impacts of the power control would be investigated under these two SNR circumstance. It is to note that with both $P_{S D}^{t}$ and $P_{L U}^{t}$ are determined by (4) for the power control case. While without power control, both LUs and SDs transmit with their defined maximum transmission power. Unless otherwise stated, the values of the other parameters are listed in Table I.

\section{A. Sensing Length}

In this subsection, we will show the energy efficiency, throughput of SD with tolerable interference to $\mathrm{LU}$ as a

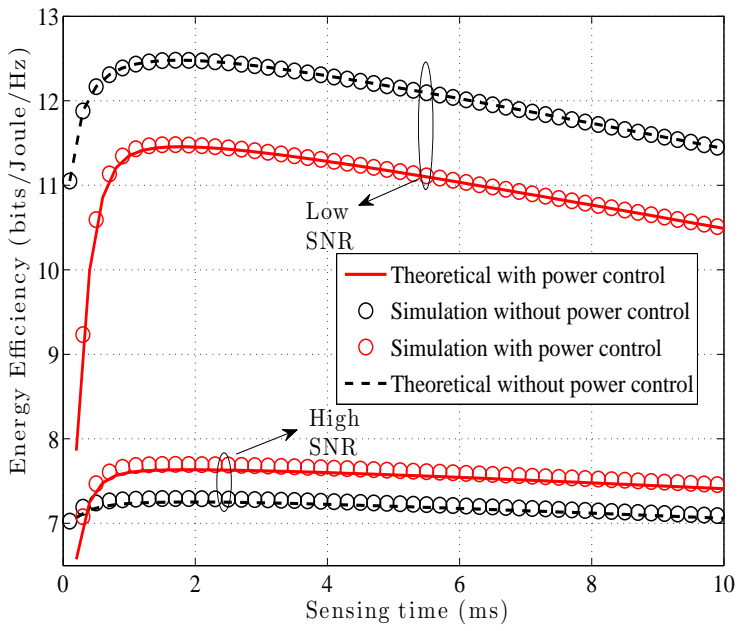

Fig. 6. Energy efficiency vs sensing time with high SNR and low SNR.

function of variable sensing length with fixed frame duration $T=100 \mathrm{~ms}$. Power control is employed in simulation to further control the interference to LU and to improve energy efficiency with $\Gamma=-50 \mathrm{~dB}$.

Fig. 5 illustrates the throughput of SD as a function of variable sensing length under high SNR (upper subfigure) and low SNR (lower subfigure) network environments, respectively. It can be clearly observed that the higher average SNR with higher maximum transmission power of SD leads to higher achievable throughput while the lower average SNR with lower maximum transmission power of SD leads to lower achievable throughput. The achievable throughput without power control under two SNR scenarios are also provided for comparison, which are always higher than the throughput with power control because of the limitation of SD transmission power. Furthermore, the optimal sensing time for the two SNR networks are quite close with each other which are at around $2.6 \mathrm{~ms}$.

In Fig. 6, the energy efficiency versus the sensing time of SD is presented for the two SNR scenarios. Compared with Fig. 5 the optimal sensing time for the maximum energy efficiency are a bit different from the optimal sensing for the maximum throughput. This is more obvious in high SNR case which has optimal sensing time for energy efficiency both located at around $5 \mathrm{~ms}$ with the power control and without power control. It is rather interesting to note that for high SNR scenario, power control leads to higher energy efficiency while power control degrades energy efficiency under low SNR network. Intuitively, due to the essence of the Shannon channel capacity formula, it should be always more energy efficient with power control because the total transmission power is lower than the case without power control. However, this only apply to the energy efficiency which is calculated as successfully transmitted information over received signal power. In order to calculate the accurate energy efficiency in practise, it can not be ignored that the 


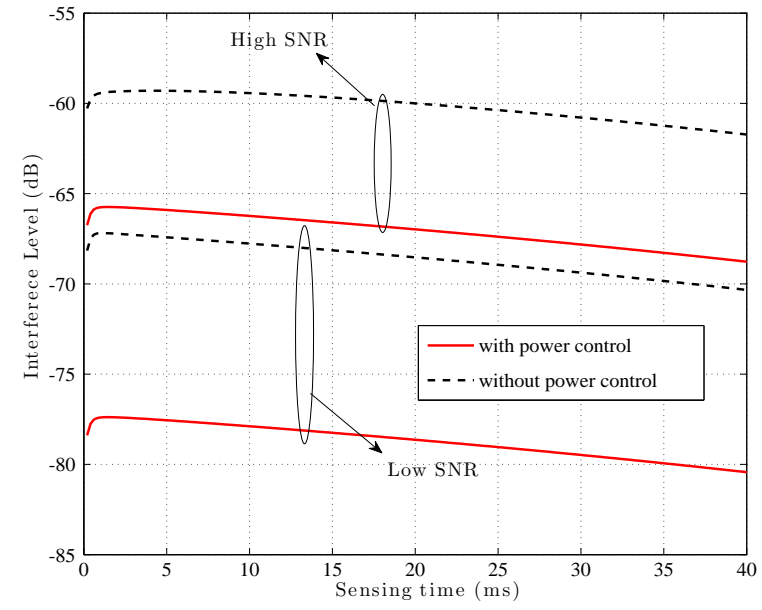

Fig. 7. Interference to LU vs sensing time with high SNR and low SNR.

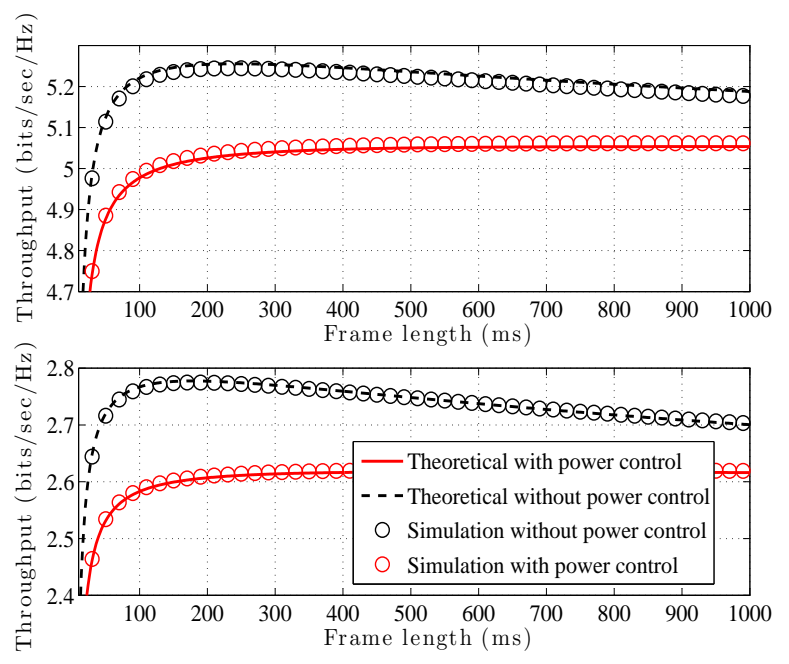

Fig. 8. Throughput vs frame length with high SNR (upper) and low SNR (lower).

impact of real channel propagation (path loss and fading) on the transmission signal power, as well as the extra power consumption such as electronic circuit power and sensing power. Specifically, under low SNR network, the transmission power is not dominant power consumption any more considering the total power consumed and this is the reason why the power control may not be energy efficient for low SNR network. In addition, the trend of caused interference with the increase in sensing length is also shown by Fig. 7. Longer sensing time provides less missed detection which causes less interference. Besides, it can be clearly illustrated that power control mitigates the interference significantly under both the SNR regimes. The impressive mitigation could reach around $6 \mathrm{~dB}$ and $10 \mathrm{~dB}$ for high SNR and low SNR networks, respectively via adaptive uplink power control.

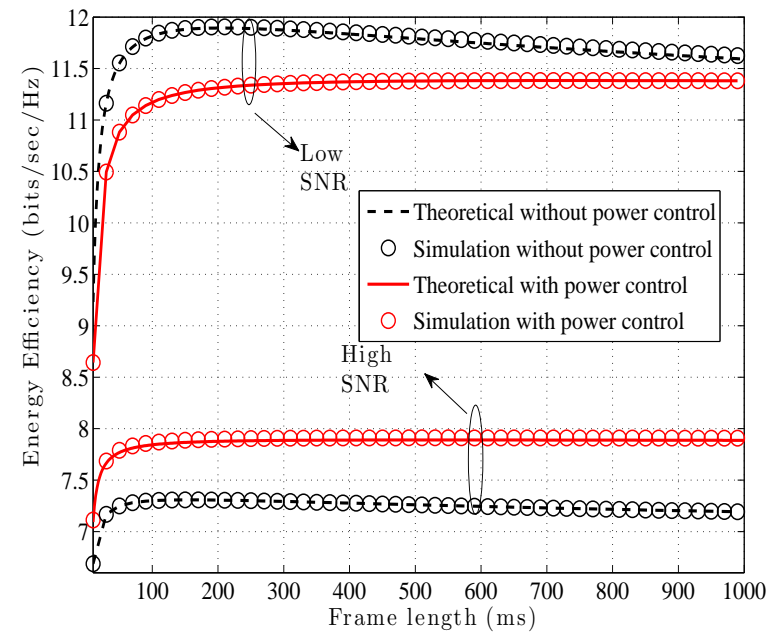

Fig. 9. Energy efficiency vs frame length with high SNR and low SNR.

\section{B. Frame Length}

In this subsection, the energy efficiency, throughput of $\mathrm{SD}$ and the interference to $\mathrm{LU}$ are presented as a function of variable frame length with fixed sensing length $\tau=2$ $\mathrm{ms}$. Same as the previous subsection, power control factor of $\mathrm{SD}$ is also set to be $\Gamma=-50 \mathrm{~dB}$.

Fig. 8 illustrates the throughput of $\mathrm{SD}$ as a function of variable frame length up to $1000 \mathrm{~ms}$ under the two network environments. It can be seen that the throughput curve without power control is similar as the previous sensing curve in Fig. 5. This is because the longer the duration of frame is, the higher probability that the LU becomes active before the current frame ends would be. In this case there is an optimal length to guarantee that it would not spend too much percentage of time on sensing at the same time the transmission would not be keeping too long in each frame which cause unnecessary interference. It can be observed that this optimal frame length under the high and low SNR regime are diverse with around $250 \mathrm{~ms}$ and $180 \mathrm{~ms}$, respectively. While the curve with power control shows a bit different trend, which keeps flat after it reaches the maximum value when $T$ approaches $500 \mathrm{~ms}$ and $400 \mathrm{~ms}$ under the corresponding SNR regime. Apparently different concavity of achievable throughputs has been shown by the power control and the without power control curves. It is to note that the reason which leads to this concavity diversity is only due to different transmission power (reduced transmission power mitigates the negative effect of long frame length on throughput) and have no direct relationship with frame length $T$. This is consistent with our discussion in section IV.

The energy efficiency of the two SNR networks are plotted versus frame length in Fig. 9. With similar trend of throughput curve, the optimal frame length can be both found at around $200 \mathrm{~ms}$ under two SNR regimes without power control. The maximum energy efficiency with power 


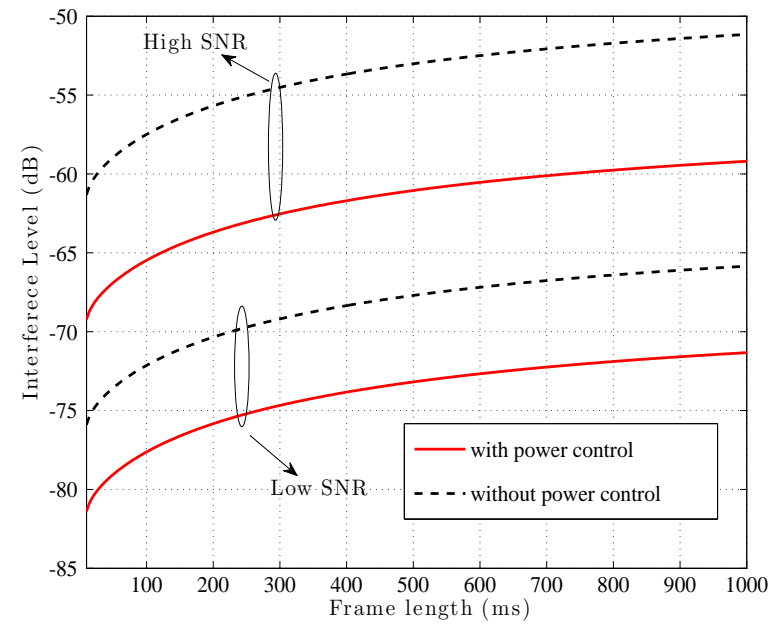

Fig. 10. Interference to LU vs frame length with high SNR and low SNR.

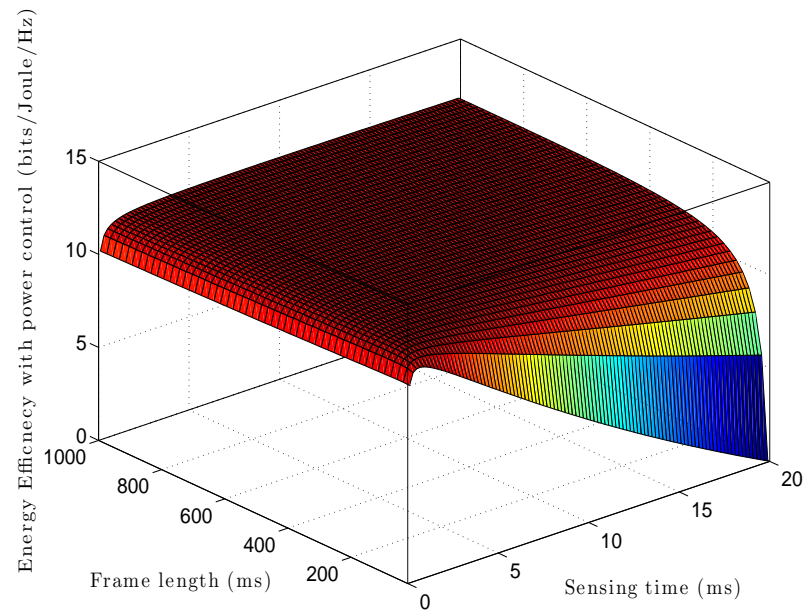

Fig. 11. Energy efficiency vs Sensing and frame length with low SNR.

control is reached a bit earlier than throughput with increase of $T$, which is located around at $200 \mathrm{~ms}$ and $350 \mathrm{~ms}$ for the high SNR and low SNR networks, respectively. The impact of power control on energy efficiency, that power control enhances energy efficiency under high SNR network while power control degrades energy efficiency under low SNR network, could be explained as the some reason as variable sensing results. Furthermore, the interference versus frame length is illustrated in Fig. 10. As a contrast to variable sensing time, the interference increases monotonously with the increase in frame length. The interference mitigation by adaptive uplink power control are around $8 \mathrm{~dB}$ under high SNR and $5.5 \mathrm{~dB}$ under low SNR. Finally, a three dimensional plot for energy efficiency under low SNR regime is shown in Fig. 11, which provides a joint insight of energy efficiency on sensing time and frame length. It can be seen that higher energy efficiency can be achieved by a

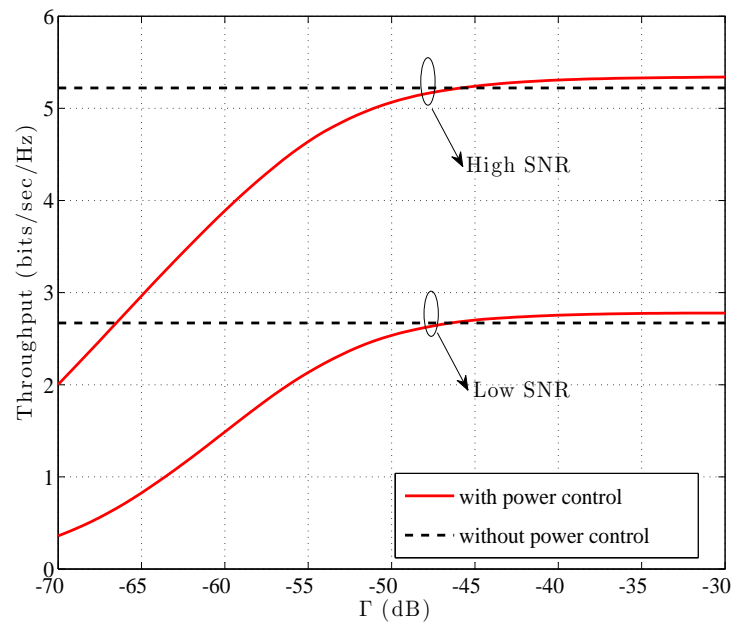

Fig. 12. Throughput vs power control factor with high SNR and low SNR.

joint appropriate selection of sensing time and frame length. Specifically, various frame lengths do not much affect the values of optimal sensing times while the performance (achievable energy efficiency) of various frame lengths is affected by the given sensing length, especially when frame length is smaller than $500 \mathrm{~ms}$. However, this effect gradually vanishes when frame length becomes larger. It is to note that the shown trend of achievable energy efficiency by various sensing time and frame length in this figure reaches consistent conclusion with our previous results.

It is also observed that the our theoretical and simulated results are in perfect agreement for all cases above.

\section{Uplink Adaptation}

Based on previous discussion, one may reach to the conclusion that the power control is not always energy efficient (for low SNR regime) in a CR aided HCN. Therefore, it would be more interesting to further explore the impact of power control of SD with variable power control factor $\Gamma$ on the energy efficiency and throughput of the proposed network. Because $P_{S D}^{0}=P_{S D}^{\max }+\Gamma$, with fixed $P_{S D}^{\max }$, it is equivalent to explore the impact of power control of SD with variable required $P_{S D}^{0}$. This may be considered as a case where the required $P_{S D}^{0}$ is varying subject to different network QoS requests. The duration of sensing and frame length are both fixed as $2 \mathrm{~ms}$ and $100 \mathrm{~ms}$, respectively. Fig. 12 illustrates the impact of variable power control factor from $\Gamma=-70 \mathrm{~dB}$ to $\Gamma=-30 \mathrm{~dB}$, on the throughput of SD. It can be seen that the throughput increases as $\Gamma$ increases which is equivalent to the increase of required $P_{S D}^{0}$ under both the low and high SNR regime during adaptive power control. There is another non-intuitive result which can be deduced from comparison of the curves with power control and without power control. That is, the achievable throughput with power control may exceed the achievable throughput without power control when $\Gamma$ is large enough. 


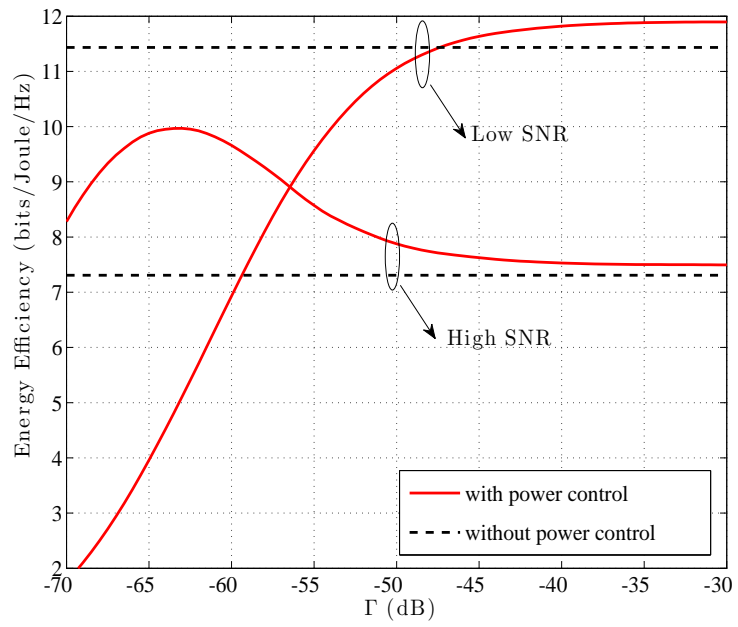

Fig. 13. Energy efficiency vs power control factor with high SNR and low SNR.

The break point is at around $-47 \mathrm{~dB}$. This is mainly because although the transmission power of SD with power control is the same as the case without power control when $\Gamma$ breaks this threshold, the LU still employs power control with fixed $\Gamma_{p}$, thereby reduces the average transmission power and benefits the achievable throughput of SD. It is implied that with targeted performance guaranteed, the LU should also employ adaptive power control in order to improve the performance of both the SD and itself.

Fig. 13 presents the energy efficiency of SD versus the variable $\Gamma$. It can be observed that under low SNR network, the energy efficiency curve has similar trend as throughput. As we discussed, the transmission power is not dominant over the total power consumption and the low received power $P_{S D}^{0}$ gives low throughput but the extra power consumptions (sensing and electronic circuit power consumption) still exist and keep the same, which degrade the energy efficiency when $\Gamma$ is relatively small. The reason why the energy efficiency is higher than the case without power control when $\Gamma$ is large enough is due to the limited transmission power of LU with power control, which is consistent with the previous throughput results we discussed before. Furthermore, it is rather interesting to investigate the impact of power control on energy efficiency under high SNR regime. It is further observed that power control would boost energy efficiency under high SNR regime because compared with high transmission power, the extra power consumptions do not affect the overall energy efficiency too much. However, the energy efficiency curve is not simply monotonously decreasing. Instead, the curve increases firstly and then decreases. This is because that even for high SNR, when $\Gamma$ is very small the transmission power of SD may be relatively small which would degrade energy efficiency like the low SNR case. As $\Gamma$ increases, the average transmission power of SD with power control also increases correspondingly and gradually becomes dominant over the total

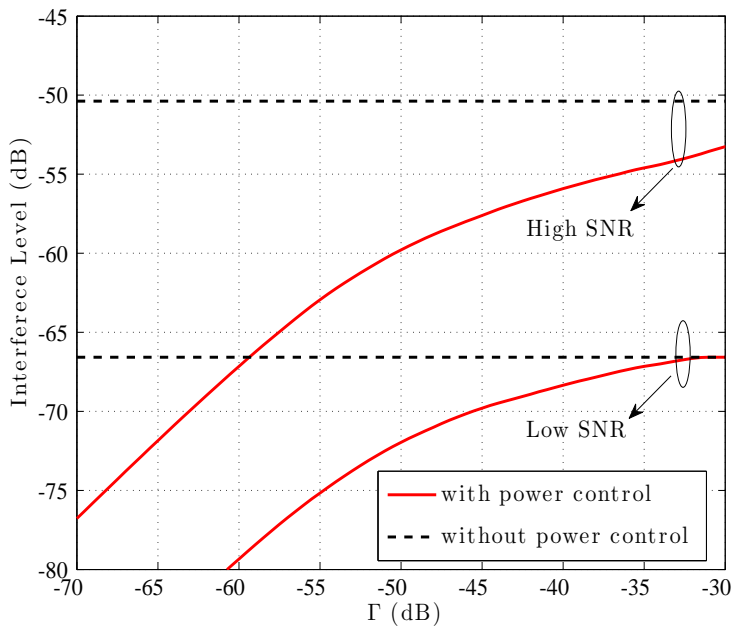

Fig. 14. Interference to LU vs power control factor with high SNR and low SNR.

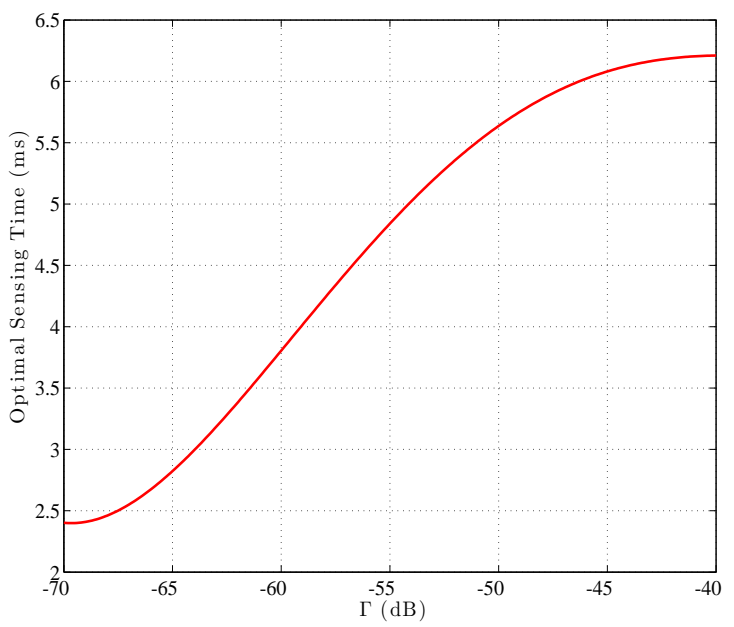

Fig. 15. Optimal sensing time for energy efficiency vs power control factor.

power consumptions. Until the energy efficiency reaches the maximum value when $\Gamma=-67 \mathrm{~dB}$ and then decreases as the transmission power approaches the case without power control. In addition, the interference to $\mathrm{LU}$ versus variable power control factor is illustrated in Fig. 14. It can be seen that under both SNR networks, as $\Gamma$ increases the interference to LU gradually increases and approaches the interference level without power control, which is consistent with our previous simulation results.

Last but not least, the impact of variable $\Gamma$ on the the optimal sensing time for the maximum energy efficiency are shown in Fig. 15. It is to note that Fig. 15 is for low SNR regime case which is almost overlapping with the curve with the high SNR regime. It can be observed that the optimal sensing increases from $2.4 \mathrm{~ms}$ to $6.2 \mathrm{~ms}$ as power control factor varies from $\Gamma=-70 \mathrm{~dB}$ to $\Gamma=-40 \mathrm{~dB}$. Moreover, 
energy efficient optimal sensing lengths and optimal frame length (the shortest length with maximum achieved energy efficiency) under two SNR regime are summarized as Table II, which also includes the results from [19] and [23]. It can be seen that the most energy efficient options of sensing and frame lengths between strategies with and without power control over high and low SNR are marked (with *) and compared with [19] and [23]. The corresponding energy efficiency gain with the optimal sensing length and frame length are highlighted in percentage, which are $11 \%$ and $14 \%$ under high SNR, and 5\% and 7\% under low SNR, respectively. It is also worthy of note that the energy efficiency gain is because of our accurate derivation model with precise optimal value and power control strategy. This table also reflects the fact that adaptive power control may lead to diversity of both the optimal sensing time and optimal frame length for the maximum energy efficiency. The sensing time, frame length and power control factor should be adaptively changed subject to the network environments to achieve the required performance.

\section{CONCLUSIONS}

In this paper, we analysed the energy efficiency and throughput of SDs in a CR aided $\mathrm{HCN}$ based on the frame structure which consists of variable sensing and data transmission slots. The achievable energy efficiency and throughput of the SD have been both analytically expressed with adaptive power control under high SNR and low SNR regimes where the mobile users (SD and LUs ) are transmitting with adaptive power to meet the desired target QoS. The impact of uplink adaptation and subsequently variable duration of sensing and frame length, on the achievable energy efficiency and throughput have been critically illustrated for both the high SNR and low SNR environments with energy detection spectrum sensing. The analysis of the tradeoff between energy efficiency and throughput of the SD is very useful to determine the future required cognitive frame structure for cellular networks. The behaviour of the adaptive power control has been investigated and it has been found that uplink power control may lead to decrease in energy efficiency of HCNs (in very low SNR regime). While power control would boost energy efficiency under high SNR regime and energy efficiency enhancement critically depends on the amount of extra power consumptions. The significant interference mitigation to LU by employing adaptive uplink power control is also verified by simulation results. Moreover, it has also been shown that the optimal sensing time and frame length which maximizes the energy efficiency of the SD strictly depends on the power control factor employed in the network. The proper frame structure and power control factor should be adaptively chosen to achieve the required performance of the SD at the same time to further reduce the interference to the legal LU.

All the current work on this issue is mainly in the scope of terrestrial cellular network. Recently, the demand for higher rate and reliable broadband communications is accelerating all over the world, which also brings attention of CR techniques into satellite communications domain to further increase system capacity, especially at Ka-band [41, 42]. As future work, we will look into possible ways and potential technical challenges of applying our current scheme into satellite-ground networks.

\section{Appendix A: Proof of Proposition 1}

The first partial derivative of variable $\hat{R}$ with respect to $T$ can be derived as

$$
\begin{aligned}
\hat{R}^{\prime}(T)= & \frac{\tau}{T^{2}}\left\{\mathscr{P}\left(\mathcal{H}_{0}\right)\left(1-\mathscr{P}_{f a}\right)\left(\hat{r}_{0}\left(1-\mathscr{P}_{p}^{s}\right)+\hat{r}_{1} \mathscr{P}_{p}^{s}\right)\right. \\
& \left.+\mathscr{P}\left(\mathcal{H}_{1}\right)\left(1-\mathscr{P}_{d}\right)\left(\hat{r}_{0}\left(1-\mathscr{P}_{i p}^{s}\right)+\hat{r}_{1} \mathscr{P}_{i p}^{s}\right)\right\} \\
& +\frac{T-\tau}{T}\left\{\mathscr{P}\left(\mathcal{H}_{0}\right)\left(1-\mathscr{P}_{f a}\right)\right. \\
& \times\left(\hat{r}_{0}\left(-\frac{\partial \mathscr{P}_{p}^{s}}{\partial T}\right)+\hat{r}_{1} \frac{\partial \mathscr{P}_{p}^{s}}{\partial T}\right) \\
& \left.+\mathscr{P}\left(\mathcal{H}_{1}\right)\left(1-\mathscr{P}_{d}\right)\left(\hat{r}_{0}\left(-\frac{\partial \mathscr{P}_{i p}^{s}}{\partial T}\right)+\hat{r}_{1} \frac{\partial \mathscr{P}_{i p}^{s}}{\partial T}\right)\right\} .
\end{aligned}
$$

The partial derivation of $\mathscr{P}_{p}^{s}$ and $\mathscr{P}_{i p}^{s}$ with respect to $T$ can be derived as

$$
\begin{aligned}
\frac{\partial \mathscr{P}_{p}^{s}}{\partial T}= & \frac{\alpha_{0}}{(T-\tau)^{2}}\left(1-\exp \left(-\frac{T-\tau}{\alpha_{0}}\right)\right) \\
& -\frac{1}{T-\tau} \exp \left(-\frac{T-\tau}{\alpha_{0}}\right), \\
\frac{\partial \mathscr{P}_{i p}^{s}}{\partial T}= & \frac{-\alpha_{1}}{(T-\tau)^{2}}\left(1-\exp \left(-\frac{T-\tau}{\alpha_{1}}\right)\right) \\
& +\frac{1}{T-\tau} \exp \left(-\frac{T-\tau}{\alpha_{1}}\right),
\end{aligned}
$$

respectively.

With series expansion of exponential function $e^{x}=1+$ $\frac{x}{1 !}+\frac{x^{2}}{2 !}+\frac{x^{3}}{3 !}+\ldots$ and when $T$ approaches $\tau$ and $+\infty$, the limit of $\mathscr{P}_{p}^{s}$ and $\mathscr{P}_{i p}^{s}$ can be derived as

$$
\begin{aligned}
\lim _{T \rightarrow \tau} \mathscr{P}_{p}^{s} & =\lim _{T \rightarrow \tau}\left(1-\frac{\alpha_{0}}{T-\tau}\left(1-\exp \left(-\frac{T-\tau}{\alpha_{0}}\right)\right)\right) \\
& =0, \\
\lim _{T \rightarrow \tau} \mathscr{P}_{i p}^{s} & =\lim _{T \rightarrow \tau} \frac{\alpha_{1}}{T-\tau}\left(1-\exp \left(-\frac{T-\tau}{\alpha_{1}}\right)\right)=1 \\
\lim _{T \rightarrow+\infty} \mathscr{P}_{p}^{s} & =\lim _{T \rightarrow+\infty}\left(1-\frac{\alpha_{0}}{T-\tau}\left(1-\exp \left(-\frac{T-\tau}{\alpha_{0}}\right)\right)\right) \\
& =1, \\
\lim _{T \rightarrow+\infty} \mathscr{P}_{i p}^{s} & =\lim _{T \rightarrow+\infty} \frac{\alpha_{1}}{T-\tau}\left(1-\exp \left(-\frac{T-\tau}{\alpha_{1}}\right)\right)=0,
\end{aligned}
$$

respectively. Similarly, the limit of $\frac{\partial \mathscr{P}_{p}^{s}}{\partial T}(T-\tau)$ and $\frac{\partial \mathscr{P}_{i p}^{s}}{\partial T}(T-\tau)$ when $T$ approaches $\tau$ and $+\infty$ can be respectively derived as 


$$
\begin{aligned}
& \Delta J=\tilde{\hat{J}}-\widetilde{J} \\
& =\frac{(T-\tau)\left(\mathscr{P}\left(\mathcal{H}_{0}\right)\left(1-\mathscr{P}_{f a}\right) \hat{r}_{0}+\mathscr{P}\left(\mathcal{H}_{1}\right)\left(1-\mathscr{P}_{d}\right) \hat{r}_{1}\right)}{P_{c} T+P_{s} \tau+P_{S D}^{t}(T-\tau)\left(\mathscr{P}\left(\mathcal{H}_{0}\right)\left(1-\mathscr{P}_{f a}\right)+\mathscr{P}\left(\mathcal{H}_{1}\right)\left(1-\mathscr{P}_{d}\right)\right)} \\
& -\frac{(T-\tau)\left(\mathscr{P}\left(\mathcal{H}_{0}\right)\left(1-\mathscr{P}_{f a}\right) r_{0}+\mathscr{P}\left(\mathcal{H}_{1}\right)\left(1-\mathscr{P}_{d}\right) r_{1}\right)}{P_{c} T+P_{s} \tau+P_{S D}^{\max }(T-\tau)\left(\mathscr{P}\left(\mathcal{H}_{0}\right)\left(1-\mathscr{P}_{f a}\right)+\mathscr{P}\left(\mathcal{H}_{1}\right)\left(1-\mathscr{P}_{d}\right)\right)}, \\
& =\frac{a c \log _{2}\left(\frac{N_{0}+h_{s s} P_{S D}^{t}}{N_{0}+h_{s s} P_{S D}^{m a x}}\right)+a(a+b)\left(P_{S D}^{\max } \log _{2}\left(1+\frac{h_{s s} P_{S D}^{t}}{N_{0}}\right)-P_{S D}^{t} \log _{2}\left(1+\frac{h_{s s} P_{S D}^{m} a x}{N_{0}}\right)\right)}{\left(c+P_{S D}^{\max }(a+b)\right)\left(c+P_{S D}^{t}(a+b)\right)} \\
& +\frac{b c \log _{2}\left(\frac{\left(N_{0}+h_{L S} P_{L U}^{t}+h_{s s} P_{S D}^{t}\right)\left(N_{0}+h_{L S} P_{L U}^{\max }\right)}{\left(N_{0}+h_{L S} P_{L U}^{\max }+h_{s s} P_{S D}^{\max }\right)\left(N_{0}+h_{L S} P_{L U}^{t}\right)}\right)}{\left(c+P_{S D}^{\max }(a+b)\right)\left(c+P_{S D}^{t}(a+b)\right)} \\
& +\frac{b(a+b)\left(P_{S D}^{\max } \log _{2}\left(1+\frac{h_{s s} P_{S D}^{t}}{N_{0}+h_{L S} P_{L U}^{t}}\right)-P_{S D}^{t} \log _{2}\left(1+\frac{h_{s s} P_{S D}^{m} a x}{N_{0}+h_{L S} P_{L U}^{\max }}\right)\right)}{\left(c+P_{S D}^{\max }(a+b)\right)\left(c+P_{S D}^{t}(a+b)\right)} . \\
& \lim _{P_{S D}^{t} \rightarrow P_{S D}^{\max }} \Delta(J)=\frac{b c \log _{2}\left(\frac{\left(N_{0}+h_{L S} P_{L U}^{t}+h_{s s} P_{S D}^{\max }\right)\left(N_{0}+h_{L S} P_{L U}^{\max }\right)}{\left(N_{0}+h_{L S} P_{L U}^{\max }+h_{s s} P_{S D}^{\max }\right)\left(N_{0}+h_{L S} P_{L U}^{t}\right)}\right)}{\left(c+P_{S D}^{\max }(a+b)\right)^{2}} \\
& +\frac{b(a+b) P_{S D}^{\max }\left(\log _{2}\left(1+\frac{h_{s s} P_{S D}^{\max }}{N_{0}+h_{L S} P_{L U}^{t}}\right)-\log _{2}\left(1+\frac{h_{s s} P_{S D}^{\max }}{N_{0}+h_{L S} P_{L U}^{\max }}\right)\right)}{\left(c+P_{S D}^{\max }(a+b)\right)^{2}}>0, \\
& \lim _{P_{S D}^{t} \rightarrow 0} \Delta(J)=\frac{a \log _{2}\left(\frac{N_{0}}{N_{0}+h_{s s} P_{S D}^{\max }}\right)+b \log _{2}\left(\frac{N_{0}+h_{L S} P_{L U}^{\max }}{N_{0}+h_{L S} P_{L U}^{\max }+h_{s s} P_{S D}^{\max }}\right)}{\left(c+P_{S D}^{\max }(a+b)\right)}<0 .
\end{aligned}
$$

$$
\begin{aligned}
& \lim _{T \rightarrow \tau} \frac{\partial \mathscr{P}_{p}^{s}}{\partial T}(T-\tau)= \\
& \lim _{T \rightarrow \tau} \frac{\alpha_{0}}{(T-\tau)}\left(1-\exp \left(-\frac{T-\tau}{\alpha_{0}}\right)\right) \\
& -\lim _{T \rightarrow \tau} \exp \left(-\frac{T-\tau}{\alpha_{0}}\right)=0, \\
& \lim _{T \rightarrow \tau} \frac{\partial \mathscr{P}_{i p}^{s}}{\partial T}(T-\tau)= \\
& \lim _{T \rightarrow \tau} \frac{-\alpha_{1}}{(T-\tau)}\left(1-\exp \left(-\frac{T-\tau}{\alpha_{1}}\right)\right) \\
& +\lim _{T \rightarrow \tau} \exp \left(-\frac{T-\tau}{\alpha_{1}}\right)=0, \\
& \lim _{T \rightarrow+\infty} \frac{\partial \mathscr{P}_{p}^{s}}{\partial T}(T-\tau)= \\
& \lim _{T \rightarrow+\infty} \frac{\alpha_{0}}{(T-\tau)}\left(1-\exp \left(-\frac{T-\tau}{\alpha_{0}}\right)\right) \\
& -\lim _{T \rightarrow+\infty} \exp \left(-\frac{T-\tau}{\alpha_{0}}\right)=0, \\
& \lim _{T \rightarrow+\infty} \frac{\partial \mathscr{P} s}{\partial T}(T-\tau)= \\
& \lim _{T \rightarrow+\infty} \frac{-\alpha_{1}}{(T-\tau)}\left(1-\exp \left(-\frac{T-\tau}{\alpha_{1}}\right)\right) \\
& +\lim _{T \rightarrow+\infty} \exp \left(-\frac{T-\tau}{\alpha_{1}}\right)=0 .
\end{aligned}
$$

By substituting the equations (A.4) - (A.11) into (A.1), the limit of $\hat{R}^{\prime}(T)$, when $T$ approaches $\tau$ and $+\infty$, can be finally expressed as

$$
\begin{aligned}
\lim _{T \rightarrow \tau} \hat{R}^{\prime}(T)= & \frac{1}{\tau}\left(\mathscr{P}\left(\mathcal{H}_{0}\right)\left(1-\mathscr{P}_{f a}\right) \hat{r}_{0}\right. \\
& \left.+\mathscr{P}\left(\mathcal{H}_{1}\right)\left(1-\mathscr{P}_{d}\right) \hat{r}_{1}\right)>0, \\
\lim _{T \rightarrow+\infty} \hat{R}^{\prime}(T)= & 0\left(\mathscr{P}\left(\mathcal{H}_{0}\right)\left(1-\mathscr{P}_{f a}\right) \hat{r}_{1}\right. \\
& \left.+\mathscr{P}\left(\mathcal{H}_{1}\right)\left(1-\mathscr{P}_{d}\right) \hat{r}_{0}\right)=0,
\end{aligned}
$$

respectively.

\section{APPENDIX B: PROOF of PROPOSITION 2}

If $\widetilde{\hat{J}}$ and $\widetilde{J}$ denote simplified energy efficiency of SD in the proposed $\mathrm{HCN}$ with power control and without power control, respectively, then $\Delta J$ can be derived as (B.1), where the parameters $\mathrm{a}, \mathrm{b}$ and $\mathrm{c}$ are given by

$$
\begin{aligned}
a & =\mathscr{P}\left(\mathcal{H}_{0}\right)\left(1-\mathscr{P}_{f a}\right), \\
b & =\mathscr{P}\left(\mathcal{H}_{1}\right)\left(1-\mathscr{P}_{d}\right), \\
c & =\frac{P_{c} T+P_{s} \tau}{T-\tau} .
\end{aligned}
$$

Then the limits of $\Delta J$ when $P_{S D}^{t}$ approaches $P_{S D}^{\max }$ and 0 can be respectively derived as (B.5) and (B.6). With the rule of $\log$ function (if $x>1$ and $y<1, \log _{x}(y)<0$ ), 
it is straightforward to achieve the results of (B.5) and (B.6). Taking (B.6) as an example, the denominator of (B.6) is positive apparently and numerator is negative because $\frac{N_{0}}{N_{0}+h_{s s} P_{S D}^{\max }}$ and $\frac{N_{0}+h_{L S} P_{L U}^{\max }}{N_{0}+h_{L S} P_{L U}^{\max }+h_{s s} P_{S D}^{\max }}$ are both $<1$. Same set of hint also applies to (B.5).

\section{REFERENCES}

1. F. C. Commission, "Spectrum policy task force report, fcc 02-155," Tech. Rep., 2002.

2. S. Haykin, "Cognitive radio: brain-empowered wireless communications," Selected Areas in Communications, IEEE Journal on, vol. 23, no. 2, pp. 201 - 220, Feb. 2005.

3. Q. Zhao and A. Swami, "A survey of dynamic spectrum access: Signal processing and networking perspectives," in Acoustics, Speech and Signal Processing, ICASSP 2007. IEEE International Conference on, vol. 4, Apr. 2007, pp. IV.1349-IV.1352.

4. I. Akyildiz, W.-Y. Lee, M. Vuran, and S. Mohanty, "A survey on spectrum management in cognitive radio networks," Communications Magazine, IEEE, vol. 46, no. 4, pp. $40-48$, Apr. 2008.

5. F. Digham, M.-S. Alouini, and M. K. Simon, "On the energy detection of unknown signals over fading channels," Communications, IEEE Transactions on, vol. 55, no. 1, pp. 21-24, 2007.

6. Y. Zeng and Y. chang Liang, "Eigenvalue-based spectrum sensing algorithms for cognitive radio," Communications, IEEE Transactions on, vol. 57, no. 6, pp. 1784 -1793 , Jun. 2009.

7. F. Penna, R. Garello, D. Figlioli, and M. Spirito, "Exact non-asymptotic threshold for eigenvalue-based spectrum sensing," in Cognitive Radio Oriented Wireless Networks and Communications, CROWNCOM '09. 4th International Conference on, Jun. 2009, pp. 1 -5.

8. T. Yucek and H. Arslan, "A survey of spectrum sensing algorithms for cognitive radio applications," Communications Surveys Tutorials, IEEE, vol. 11, no. 1, pp. 116 -130, Mar. 2009.

9. M. Shakir, W. Tang, A. Rao, M. Imran, and M.-S. Alouini, "Eigenvalue ratio detection based on exact moments of smallest and largest eigenvalues," in Cognitive Radio Oriented Wireless Networks and Communications (CROWNCOM), 2011 Sixth International ICST Conference on, Jun. 2011, pp. $46-50$.

10. M. Shakir, A. Rao, and M.-S. Alouini, "Collaborative spectrum sensing based on the ratio between largest eigenvalue and geometric mean of eigenvalues," in GLOBECOM Workshops (GC Wkshps), IEEE, Dec. 2011, pp. 913 -917.

11. I. Mitola, J. and J. Maguire, G.Q., "Cognitive radio: making software radios more personal," Personal Communications, IEEE, vol. 6, no. 4, pp. 13 -18, Aug. 1999.

12. V. Chair, "Cool cellular - energy efficient network architectures and transmission methods," avaiable at http://www.vodafonechair.com/research/projects cool cellular.html.
13. C. Anderson, D. Freeman, I. James, A. Johnston and S. Ljung, Mobile Media and Applications, From concept to Cash: Successful Service Creation and Launch. Wiley, 2006.

14. M. Z. Shakir, K. A. Qaraqe, H. Tabassum, M. S. Alouini, E. Serpedin, and M. A. Imran, "Green heterogeneous small-cell networks: toward reducing the co2 emissions of mobile communications industry using uplink power adaptation," IEEE Communications Magazine, vol. 51, no. 6, pp. 52-61, Jun. 2013.

15. G. Fodor, E. Dahlman, G. Mildh, S. Parkvall, N. Reider, G. Miklos, and Z. Turanyi, "Design aspects of network assisted device-to-device communications," Communications Magazine, IEEE, vol. 50, no. 3, pp. 170-177, Mar. 2012.

16. K. Doppler, M. Rinne, C. Wijting, C. Ribeiro, and K. Hugl, "Device-to-device communication as an underlay to lte-advanced networks," Communications Magazine, IEEE, vol. 47, no. 12, pp. 42-49, Dec 2009.

17. A. Hamdi, H. Tabassum, E. Hossain, and D. Kim, "Cognitive spectrum access in device-to-device enabled cellular networks," Communications Magazine, IEEE, May 2015.

18. Y.-C. Liang, Y. Zeng, E. Peh, and A. T. Hoang, "Sensing-throughput tradeoff for cognitive radio networks," in Communications, 2007. ICC '07. IEEE International Conference on, Jun. 2007, pp. 5330 -5335.

19. — - "Sensing-throughput tradeoff for cognitive radio networks," Wireless Communications, IEEE Transactions on, vol. 7, no. 4, pp. 1326 -1337, Apr. 2008.

20. Y. Pei, Y.-C. Liang, K. C. Teh, and K. H. Li, "Sensingthroughput tradeoff for cognitive radio networks: A multiple-channel scenario," in Personal, Indoor and Mobile Radio Communications, 2009 IEEE 20th International Symposium on, Sep. 2009, pp. 1257 -1261.

21. S. Stotas and A. Nallanathan, "Optimal sensing time and power allocation in multiband cognitive radio networks," Communications, IEEE Transactions on, vol. 59, no. 1, pp. 226 -235, Jan. 2011.

22. Y. Pei, A. T. Hoang, and Y.-C. Liang, "Sensingthroughput tradeoff in cognitive radio networks: How frequently should spectrum sensing be carried out?" in Personal, Indoor and Mobile Radio Communications, 2007. PIMRC 2007. IEEE 18th International Symposium on, Sep. 2007, pp. $1-5$.

23. L. Li, X. Zhou, H. Xu, G. Li, D. Wang, and A. Soong, "Energy-efficient transmission in cognitive radio networks," in Consumer Communications and Networking Conference (CCNC), 2010 7th IEEE, Jan. 2010, pp. 1 -5 .

24. Y. Pei, Y.-C. Liang, K. C. Teh, and K. H. Li, "Energyefficient design of sequential channel sensing in cognitive radio networks: Optimal sensing strategy, power allocation, and sensing order," Selected Areas in Communications, IEEE Journal on, vol. 29, no. 8, pp. 1648 -1659 , Sep. 2011. 
25. Y. Chen, G. Yu, Z. Zhang, H.-H. Chen, and P. Qiu, "On cognitive radio networks with opportunistic power control strategies in fading channels," Wireless Communications, IEEE Transactions on, vol. 7, no. 7, pp. 2752-2761, 2008.

26. R. Zhang, "Optimal power control over fading cognitive radio channel by exploiting primary user csi," in Global Telecommunications Conference, 2008. IEEE GLOBECOM 2008. IEEE, 2008, pp. 1-5.

27. A. T. Hoang, Y.-C. Liang, and M. Islam, "Power control and channel allocation in cognitive radio networks with primary users' cooperation," Mobile Computing, IEEE Transactions on, vol. 9, no. 3, pp. 348-360, 2010.

28. G. Fodor, E. Dahlman, G. Mildh, S. Parkvall, N. Reider, G. Miklos, and Z. Turanyi, "Design aspects of network assisted device-to-device communications," Communications Magazine, IEEE, vol. 50, no. 3, pp. 170-177, Mar. 2012.

29. H. Min, J. Lee, S. Park, and D. Hong, "Capacity enhancement using an interference limited area for deviceto-device uplink underlaying cellular networks," Wireless Communications, IEEE Transactions on, vol. 10, no. 12, pp. 3995-4000, Dec. 2011.

30. C.-H. Yu, K. Doppler, C. Ribeiro, and O. Tirkkonen, "Resource sharing optimization for device-to-device communication underlaying cellular networks," Wireless Communications, IEEE Transactions on, vol. 10, no. 8, pp. 2752-2763, Aug. 2011.

31. C. An, P. Si, and H. Ji, "Wideband spectrum sensing scheme in cognitive radio networks with multiple primary networks," in Wireless Communications and Networking Conference (WCNC), 2011 IEEE, Mar. 2011, pp. $67-71$.

32. H. Urkowitz, "Energy detection of unknown deterministic signals," Proceedings of the IEEE, vol. 55, no. 4, pp. 523-531, 1967.

33. K. Arshad, M. A. Imran, and K. Moessner, "Collaborative spectrum sensing optimisation algorithms for cognitive radio networks," Digital Multimedia Broadcasting, International Journal of, vol. 2010, no. Article ID 424036, 2010.

34. A. Ghasemi and E. Sousa, "Opportunistic spectrum access in fading channels through collaborative sensing," Communications, Journal of, vol. 2, no. 2, 2007.

35. Andrea Goldsmith, Wireless Communications. Cambridge University Press, New York, 2003.

36. IEEE.802.22, "Working group on wireless regional area networks," Available at http://ieee802.org/22/, 2011.

37. M.-S. Alouini and A. Goldsmith, "Area spectral efficiency of cellular mobile radio systems," Vehicular Technology, IEEE Transactions on, vol. 48, no. 4, pp. 1047-1066, 1999.

38. 3GPP, "The 3rd generation partnership project: Technical specification group radio access network; user equipment (ue) radio transmission and reception (tdd)," Available at http://www.quintillion.co.jp/3GPP/Specs/25102910.pdf, 2010.

39. W. Tang, M. Shakir, M. Imran, R. Tafazolli, and M.S. Alouini, "Throughput analysis for cognitive radio networks with multiple primary users and imperfect spectrum sensing," Communications, IET, vol. 6, no. 17, pp. 2787-2795, 2012.

40. T. Persson, C. Trnevik, L.-E. Larsson, and J. Lovn, "Output power distributions of terminals in a $3 \mathrm{G}$ mobile communication network," Bioelectromagnetics, vol. 33, no. 4, pp. 320-325, 2012. [Online]. Available: http://dx.doi.org/10.1002/bem.20710

41. W. Tang, P. Thompson, and B. Evans, "Frequency sharing between satellite and terrestrial systems in the ka band: A database approach," in Communications (ICC), 2015 IEEE International Conference on, Jun. 2015, pp. 867-872.

42. W. Tang, P. Thompson, A. Kyrgiazos, and B. Evans, "Extending the usable ka-band spectrum for FSS satellite systems by using a FS database," International Journal on Advances in Telecommunications, vol. 8, no. 3-4, 2015. 
TABLE I

Simulation Parameter Settings

\begin{tabular}{|c|c|}
\hline Narrowband bandwidth $(B)$ & $6 \mathrm{MHz}$ \\
\hline Noise PSD $\left(N_{0} / 2\right)$ & $-198 \mathrm{dBW} / \mathrm{Hz}$ \\
\hline HCN cell radius $\left(r_{d}\right)$ & $500 \mathrm{~m}$ \\
\hline Circuit power $\left(P_{c}\right)$ & $200 \mathrm{~mW}$ \\
\hline Sensing power $\left(P_{s}\right)$ & $100 \mathrm{~mW}$ \\
\hline Worst-case received SNR from LU & $-15 \mathrm{~dB}$ \\
\hline PC factor of LU $\left(\Gamma_{p}\right)$ & $-60 \mathrm{~dB}$ \\
\hline Target detection probability $\left(\overline{\mathscr{P}}_{d}\right)$ & $90 \%$ \\
\hline Mean of traffic and idle duration of LU $\left(\alpha_{1}, \alpha_{0}\right)$ & $(352 \mathrm{~ms}$, \\
& $650 \mathrm{~ms})$ \\
\hline Basic path-loss exponent $\left(\beta_{a}\right)$ & 2 \\
\hline Additional path-loss exponent $\left(\beta_{b}\right)$ & 2 \\
\hline Break point of path-loss curve $(g)$ & $1212 \mathrm{~m}$ \\
\hline Path-loss constant $(K)$ & 1 \\
\hline
\end{tabular}

TABLE II

ENERGY EFFICIENT OPTIMAL SENSING LENGTH AND FRAME LENGTH (* STAND FOR THE MOST ENERGY EFFICIENT OPTION BETWEEN WITH AND WITHOUT POWER CONTROL UNDER HIGH AND LOW SNR)

\begin{tabular}{|c|c|c|c|c|}
\hline & \multicolumn{2}{|c|}{ High SNR } & \multicolumn{2}{c|}{ Low SNR } \\
\hline & Sensing length & Frame length & Sensing length & Frame length \\
\hline With power control & $1.8 \mathrm{~ms}$ & $366 \mathrm{~ms}^{*}$ & $1.8 \mathrm{~ms}$ & $396 \mathrm{~ms}$ \\
\hline Without power control & $1.8 \mathrm{~ms}$ & $132 \mathrm{~ms}$ & $1.6 \mathrm{~ms} *$ & $160 \mathrm{~ms}^{*}$ \\
\hline Results from [19] and [23] & $2.55 \mathrm{~ms}$ & $100 \mathrm{~ms}$ & $2.55 \mathrm{~ms}$ & $100 \mathrm{~ms}$ \\
\hline Energy efficiency gain between [19]/[23] and * & $11 \%$ & $14 \%$ & $5 \%$ & $7 \%$ \\
\hline
\end{tabular}

